\title{
$\beta$-catenin activation drives thymoma initiation and progression in mice
}

\author{
Chih-Chia Liang1, Tsai-Ling Lu' ${ }^{1}$, Yi-Ru Yu ${ }^{1}$, Li-Ru You ${ }^{2,3}$ and Chun-Ming Chen ${ }^{1,3,4}$ \\ ${ }^{1}$ Department of Life Sciences and Institute of Genome Sciences, National Yang-Ming University, Taipei, Taiwan \\ ${ }^{2}$ Institute of Biochemistry and Molecular Biology, National Yang-Ming University, Taipei, Taiwan \\ ${ }^{3}$ VYM Genome Research Center, National Yang-Ming University, Taipei, Taiwan \\ ${ }^{4}$ Taiwan Mouse Clinic-National Phenotyping Center, Taipei, Taiwan \\ Correspondence to: Chun-Ming Chen, email: cmchen@ym.edu.tw
}

Keywords: thymic epithelium, thymus, $\beta 5$ t, cytokeratin 5, p63, AIRE

Received: February 23, $2015 \quad$ Accepted: June 01, $2015 \quad$ Published: June 08, 2015

This is an open-access article distributed under the terms of the Creative Commons Attribution License, which permits unrestricted use, distribution, and reproduction in any medium, provided the original author and source are credited.

\section{ABSTRACT}

Thymoma is the most commonly identified cancer in the anterior mediastinum. To date, the causal mechanism that drives thymoma progression is not clear. Here,

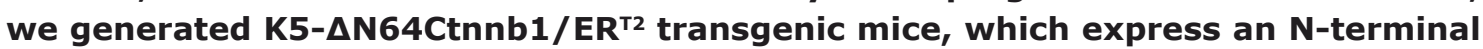
deletion mutant of $\beta$-catenin fused to a mutated ligand-binding domain of estrogen receptor $\left(E R^{\mathrm{T} 2}\right)$ under the control of the bovine cytokeratin 5 (K5) promoter. The transgenic mouse lines named Tg1 and Tg4 were characterized. Forced expression

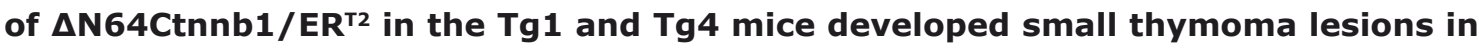
response to tamoxifen treatment. In the absence of tamoxifen, the $\mathrm{Tg} 1$ mice exhibited leaky activation of $\beta$-catenin, which activated the TOP-Gal transgene and $\mathrm{Wnt} / \boldsymbol{\beta}$ catenin-targeted genes. As the Tg1 mice aged in the absence of tamoxifen, manifest thymomas were found at 10-12 months. Interestingly, we detected loss of AIRE and increase of p63 in the thymomas of $\mathrm{Tg} 1$ mice, similar to that observed in human thymomas. Moreover, the $\beta 5 t$ protease subunit, which was reported as a differential marker for human type B3 thymoma, was expressed in the Tg1 thymomas. Thus, the Tg1 mice generated in this study accurately mimic the characteristics of human thymomas and may serve as a model for understanding thymoma pathogenesis.

\section{INTRODUCTION}

The thymus is an organ that provides a specialized microenvironment for the thymocyte development. The thymic microenvironment consists of a variety of cell types, including the thymic epithelial cells (TECs). TECs can be further anatomically sub-categorized as cortical TECs (cTECs) and medullary TECs (mTECs). cTECs are essential for the positive selection of developing thymocytes, while mTECs establish self-tolerance of selected thymocytes [1]. cTECs and mTECs can be distinguished by differentially expressed cytokeratin profiles in the adult human thymus [2]. In mice, cytokeratin 8 (K8) and cytokeratin 5 (K5) are also commonly used for histological examination of TEC populations in the cortex and medulla of the postnatal thymus [3-6]. In addition, mTECs express p63 [6,7],
AIRE [6, 8-12] and UEA-1 [5, 13-15], whereas cTECs express $\beta 5 t[12,16,17]$ and Ly51 $[6,12]$; these additional markers can be used to further sub-classify $\mathrm{EpCAM}^{+} \mathrm{TEC}$ populations in the physiological or pathological thymus.

Tumors arising from TECs are called thymomas or thymic carcinomas and account for $0.2 \%-1.5 \%$ of all cancers types $[18,19]$. Classification of thymic epithelial tumors is mainly based on the histological features of the tumor, including epithelial cell morphology, rich or scant thymocytes, cellular atypia, and invasiveness. Due to the rarity of thymic epithelial tumors, the etiological factors that contribute to the development of thymomas remain unclear.

According to the World Health Organization (WHO), the histological classification of thymic epithelial tumors is subdivided into type A thymomas, type B thymomas, type $\mathrm{AB}$ mixed, and type $\mathrm{C}$ (or thymic carcinoma) [20]. The 
type A thymoma is comprised of spindle or oval neoplastic cells with few or no lymphocytes. Type B thymoma has predominantly large polygonal or round epithelial cells. Type B thymomas can be further subdivided into types B1, B2, or B3 based on the morphology of neoplastic epithelial cells and the extent of lymphocytic infiltration. The type B1 thymoma is a lymphocyte-rich tumor, and the type B2 thymoma exhibits an increased number of clustered or scattered neoplastic epithelial cells among a large population of infiltrated lymphocytes. The type B3 thymoma is composed of predominantly epithelial cells with few or no lymphocytes. In addition, an unique perivascular space pattern is commonly present in the types $\mathrm{B} 2$ and $\mathrm{B} 3$ thymomas. Type $\mathrm{AB}$ mixed thymoma is similar to type A thymoma with lymphocyte-rich areas. Type $\mathrm{C}$ thymoma (or thymic carcinoma) is a rare tumor type that displays higher invasiveness and metastatic potential [18]. The WHO classification system appears to reflect the oncogenic potential of thymomas and correlates with the clinical stages of patients with thymoma [21, 22]; however, the small number of cases has impeded the ability to establish statistical significance between the WHO histological typing system and clinical outcomes [23]. Therefore, further studies on the biological behavior, histopathological features, prognosis, therapeutic strategies, and follow-up information of thymomas are highly warranted.

Previous molecular genetic studies have identified loss of heterozygosity $(\mathrm{LOH})$ at the adenomatous polyposis coli $(A P C)$ locus was associated with the type $\mathrm{B}$ thymomas $[24,25]$. The critical role of APC-mediated signaling in thymic epithelial differentiation and carcinogenesis was further explored in mice by conditional ablation of the Apc gene using K14-Cre [26]. Interestingly, loss of Apc in K14-expressing TECs caused thymic atrophy and squamous metaplasia, suggesting that $A p c$ is required for thymic epithelial differentiation and development [26]. APC is known to play a key role in regulating stability and nuclear entry of $\beta$-catenin, which is a crucial mediator of the canonical Wnt signaling pathway involved in cellfate determination, proliferation, embryonic development, and cancer progression [27]. Forced expression of stabilized $\beta$-catenin in embryonic Foxn1-expressing thymic epithelial primordium leads to the loss of thymic epithelial identities and transdifferentiates into epidermal cell-fate [28]. However, the thymoma phenotype was not described in either study $[26,28]$. Defects in early thymic organogenesis likely preclude the long-term monitoring of thymoma development.

In our previous study, we demonstrated that loss of $\beta$-catenin in a $\mathrm{K} 5$-expressing TEC lineage resulted in thymic atrophy [6]. Loss of $\beta$-catenin leads to aberrant differentiation of TECs and subsequent thymocyte development defects [6]. Thus, $\beta$-catenin levels may be critical for the physiological and pathological states of TECs. To examine the role of $\beta$-catenin in thymic homeostasis, we generated an inducible transgenic mouse named $K 5-\Delta N 64 C t n n b 1 / E R^{T 2}$, which expresses the $C$-terminal portion of $\beta$-catenin fused to a mutated ligand-binding domain of the estrogen receptor $\left(\mathrm{ER}^{\mathrm{T} 2}\right)$ to control activation of $\beta$-catenin in K5-expressing cells using tamoxifen (Tam). Our results with this transgenic line show that $\Delta \mathrm{N} 64 \mathrm{Ctnnb} 1 / \mathrm{ER}^{\mathrm{T} 2}$ can be induced by Tam treatment in both TECs and hair follicles. Very importantly, we observed that nuclear localization of $\beta$-catenin in TECs promoted thymoma progression. These results indicate that $\beta$-catenin is critical for maintaining thymic epithelial homeostasis and driving thymoma progression.

\section{RESULTS}

\section{Characterization of transgenic mice expressing $\triangle \mathrm{N} 64 \mathrm{Ctnnb} 1 / \mathrm{ER}^{\mathrm{T} 2}$ driven by bovine $\mathrm{K5}$ promoter in the thymus and skin}

To address the function of stabilized $\beta$-catenin in the thymic epithelium of adult mice, K5- $\Delta$ N64Ctnnb1/ $E R^{T 2}$ mice were generated. These mice harbor an $\mathrm{N}$-terminal, 64-amino-acid truncation of $\beta$-catenin fused to the tamoxifen (Tam)-inducible ligand-binding domain of estrogen receptor $\left(E R^{T 2}\right)$ driven by a bovine $\mathrm{K} 5$ promoter (Figure 1A). The expression of $\triangle \mathrm{N} 64 \mathrm{Ctnnb} 1 /$ $\mathrm{ER}^{\mathrm{T} 2}$ in various organs of transgenic mouse lines 1,4 , and 10 (hereafter referred to $\operatorname{Tg} 1, \operatorname{Tg} 4$, and $\operatorname{Tg} 10$ ) were detected using an anti- $\beta$-Catenin antibody for western blot analysis. We found that $\mathrm{Tg} 1$ and $\mathrm{Tg} 4$ exhibited comparable amounts of the $\Delta \mathrm{N} 64 \mathrm{Ctnnb} 1 / \mathrm{ER}^{\mathrm{T} 2}$ fusion protein in the skin, whereas skin from $\mathrm{Tg} 10$ expressed lower levels of $\Delta \mathrm{N} 64 \mathrm{Ctnnb} 1 / \mathrm{ER}^{\mathrm{T} 2}$ fusion protein (Figure 1B). In the thymus and the esophagus, we observed higher $\Delta \mathrm{N} 64 \mathrm{Ctnnb} 1 / \mathrm{ER}^{\mathrm{T} 2}$ protein levels in the $\mathrm{Tg} 1$ mice than in $\mathrm{Tg} 4$ or $\mathrm{Tg} 10$ mice (Figure 1B). In general, $\triangle \mathrm{N} 64 \mathrm{Ctnnb} 1 / \mathrm{ER}^{\mathrm{T} 2}$ expression of $\mathrm{Tg} 10$ was lowest among three transgenic lines (Figure 1B). We further examined transgene copy numbers of Tg1 and Tg4 mice using southern blot analysis. We found that Tg1 and Tg4 carried about 8 and 4 copies of the transgene, respectively (Supplementary Figure 1). Thus, Tg1 and Tg4, which had higher transgene expression, were selected for more detailed characterization.

Furthermore, we characterized $\Delta \mathrm{N} 64 \mathrm{Ctnnb} 1 / \mathrm{ER}^{\mathrm{T} 2}$ expression and activation in the TECs of the Tg1 mice at 8 weeks of age. After 3 days of Tam administration, thymi were collected and TECs were sorted. Within $\mathrm{CD}^{-}{ }^{-} \mathrm{EpCAM}^{+}$gated TECs, mTECs and cTECs were analyzed and sorted using cell surface markers UEA1 and Ly51, respectively (Supplementary Figure 2A). We found that the percentages of mTECs $\left(\mathrm{UEA}^{+}\right)$and cTECs $\left(\mathrm{Ly}_{51^{+}}\right)$in $\mathrm{CD}^{-} 5^{-} \mathrm{EpCAM}^{+}$gated cells were 

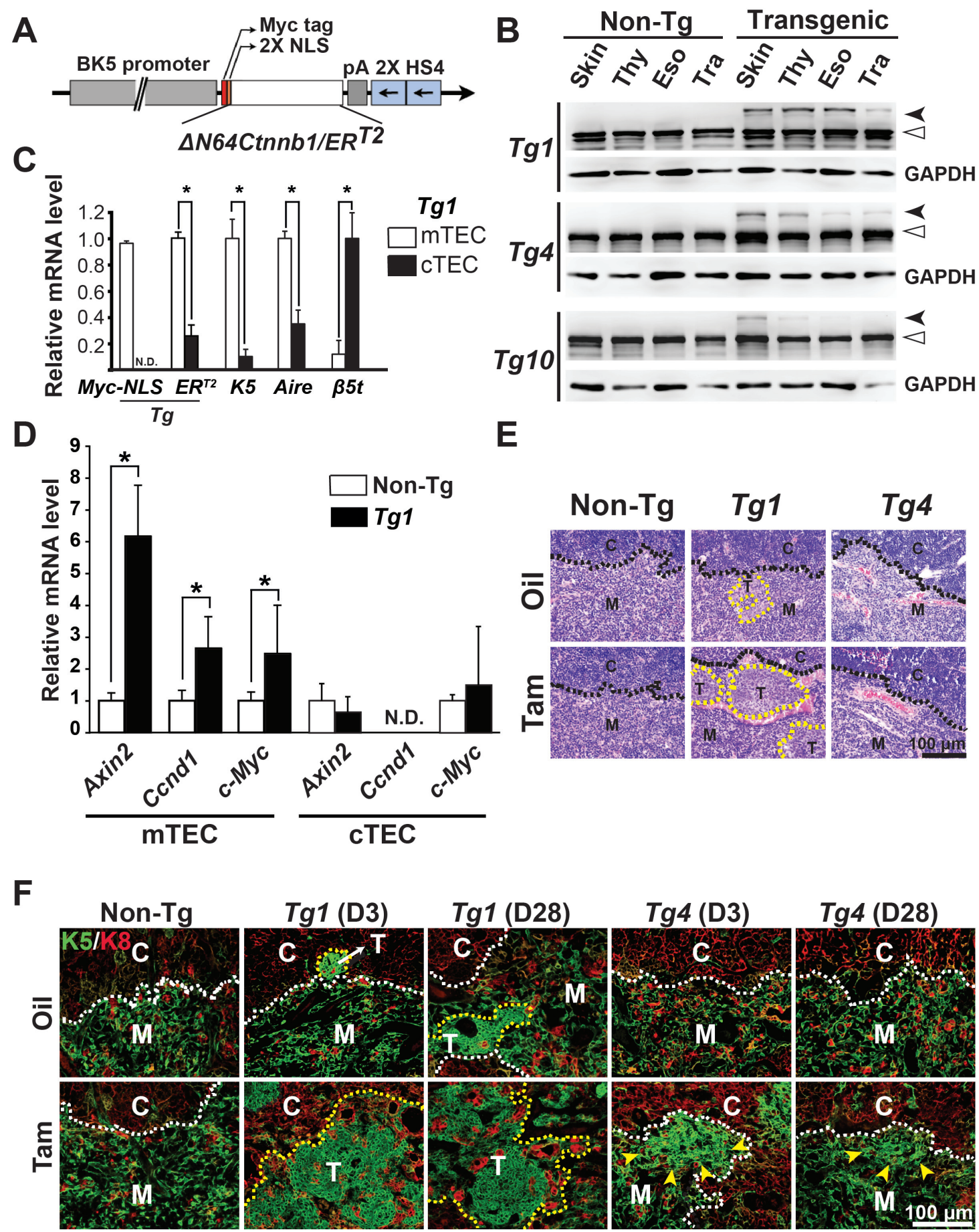

Figure 1: Characterization of $\operatorname{Tg}\left(K 5-\Delta N 64-C t n n b 1 / E R^{T 2}\right)$ transgenic mice. A. Diagram illustrates the construct used for generating the transgenic mice. B. Western blot analysis reveals relative expression levels of endogenous $\beta$-catenin (open arrowheads) and transgenic $\Delta \mathrm{N} 64-\mathrm{Ctnnb} 1 / \mathrm{ER}^{\mathrm{T} 2}$ fusion protein (close arrowheads) in various organs (skin, thymus, esophagus, and trachea) of Tg1, Tg4, and Tg10 compared to non-transgenic (non-Tg) controls. GAPDH served as an internal control. C. Relative expression levels of $\Delta N 64-C t n n b 1 /$ $E R^{\mathrm{T} 2}$ transgene (Myc-NLS and ER ${ }^{\mathrm{T} 2}$ ), mTEC-specific $K 5$ and Aire, and cTEC-specific $\beta 5 t$ expression are determined by RT-qPCR. RNA samples were isolated from the sorted CD45-EpCAM+UEA1 ${ }^{+}$mTECs and CD45-EpCAM ${ }^{+} \operatorname{Ly} 51^{+}$cTECs of Tg1 thymi. N.D., not detected. D. Relative expression levels of Axin2, Ccnd1, and c-Myc are determined by RT-qPCR. RNA was isolated from the sorted mTECs and cTECs of non-Tg or Tg1 thymi. E. H\&E staining of thymic sections from vehicle (oil)- and Tam-treated non-Tg, Tg1, and Tg4 mice shows notable cortex and medulla areas, referred to as $\mathrm{C}$ and $\mathrm{M}$ (separated by black dashed lines). Aberrant lesions in the Tg1 thymic sections are depicted by yellow dashed lines at the cortical-medullary junction (CMJ). F. Co-immunofluorescent staining of K5 (green) and K8 (red) in the thymic sections from oil- and Tam-treated non-Tg, Tg1, and Tg4 mice for 3 or 28 days shows cTECs at the cortex C., mTECs at medulla (M) (separated by white dashed lines), and aberrant K5-expressing TEC clusters (T; depicted by yellow dashed lines or pointed by arrowheads). Scale bar, $100 \mu \mathrm{m}$. 
comparable between non-transgenic control (non-Tg) and Tg1 thymi (Supplementary Figure 2B). Using RT-qPCR, we found that $\Delta \mathrm{N} 64 \mathrm{Ctnnb} 1 / \mathrm{ER}^{\mathrm{T} 2}$ expression detected by the transgene-specific primers (Myc-NLS or ER ${ }^{\mathrm{T} 2}$ ) was significantly enriched in CD45-EpCAM ${ }^{+} \mathrm{UEA}^{+}{ }^{+}$mTECs (Figure 1C). As expected, the mTEC-specific genes, K5 and Aire, and cTEC-specific $\beta 5 \mathrm{t}$ were enriched in the $\mathrm{CD}^{-}{ }^{-} \mathrm{EpCAM}^{+} \mathrm{UEA}^{+}{ }^{+}$mTECs and CD45-EpCAM ${ }^{+} \mathrm{Ly}^{-} 1^{+}$ cTECs, respectively (Figure 1C). In consistent with the transgene expression, we also found that Wnt/ $\beta$-catenintargeted genes, such as Axin2, cyclin D1, and c-Myc, were significantly upregulated in the mTECs, but not in the cTECs, of Tam-treated Tg1 mice (Figure 1D). These data suggest that the $\triangle \mathrm{N} 64 \mathrm{Ctnnb} 1 / \mathrm{ER}^{\mathrm{T} 2}$ transgene, driven by $\mathrm{K} 5$ promoter, is restricted and can be induced by Tam to activate its target genes in mTECs.

Next, we examined several potential phenotypes of TECs using histology and K5/K8 co-immunofluorescence analyses in the presence or absence of Tam administration to 8 -week-old Tg1 and Tg4 mice. Microscopically, we found a few small lesions with squamoid appearance and rare lymphocytes in the thymic cortical-medullary junction (CMJ) of vehicle-treated Tg1 mice. These lesions were expanded in the Tam-treated Tg1 thymic CMJ and medulla (Figure 1E, middle). However, we found no overt phenotype in the histological views of thymic medullae from vehicle only (oil-treated) or Tam-treated Tg4 or nonTg control mice (Figure 1E). We performed K5/K8 coimmunostaining on the thymic sections of non-Tg control, $\mathrm{Tg} 1$, and $\mathrm{Tg} 4$ mice after vehicle or Tam treatment for 3 or 28 days. In the non-Tg control, we observed K5- and K8expressing cells predominately in the medulla and cortex, respectively (Figure 1E). In the vehicle-treated Tg1 mice, aberrant $\mathrm{K} 5$-expressing cell clusters residing in the CMJ of the thymi were observed (Figure 1F), indicating possible leaky expression of -catenin, leading to sufficient activity to drive thymoma initiation. However, in the vehicletreated Tg4 mice, the aberrant K5-expressing cell clusters were not detected (Figure 1F). After Tam treatment for 3 days or 28 days in Tg1 and Tg4 mice, expanded K5expressing squamoid cells were observed, although the aberrantly expanded K5-expressing lesions were smaller in the thymi of Tg4 (Figure 1F). This observation may be due to lower $\Delta \mathrm{N} 64 \mathrm{Ctnnb} 1 / \mathrm{ER}^{\mathrm{T} 2}$ transgene expression in the thymus as seen by western blot (Figure 1B).

Next, we examined $\Delta$ N64Ctnnb1/ER ${ }^{\mathrm{T} 2}$ expression in hair cycle re-entry because previous studies have demonstrated that transient $\beta$-catenin activation in the skin results in stimulation of resting (telogen) hair follicles to the growing state (anagen phase) [29, 30]. Therefore, we applied Tam topically on the back skin of Tg1 and Tg4 mice and examined hair cycle re-entry. In agreement with these reports [29, 30], we observed that hair follicles had grown into deeper layers of the dermis, with marked expansion of outer root sheet (ORS) cells, in Tg1 and Tg4 mice that received Tam compared to those that received vehicle (EtOH) only (Supplementary Figure 3A). We also examined potential leakage activation of $\Delta \mathrm{N} 64 \mathrm{Ctnnb} 1 /$ $\mathrm{ER}^{\mathrm{T} 2}$ fusion protein in the absence of Tam. We observed darker skin, indicating hair follicles in growing state, on the back skin of shaved Tg1 and Tg4 mice compared to non- $\mathrm{Tg}$ controls without Tam treatment for 16-20 days (Supplemental Figure 3B), suggesting possible leaky expression and activity of $\beta$-catenin. Taken together, our data suggest that $\triangle \mathrm{N} 64 \mathrm{Ctnnb} 1 / \mathrm{ER}^{\mathrm{T} 2}$ can be induced by Tam treatment in both TECs and hair follicles in Tg mice, although leaky expression of active $\Delta \mathrm{N} 64 \mathrm{Ctnnb} 1 / \mathrm{ER}^{\mathrm{T} 2}$ may be present.

\section{Monitoring thymomas in leakage activation of $\beta$-catenin in K5-expressing TECs in the aged transgenic mice}

Because leaky expression of active $\Delta$ N64Ctnnb1/ $\mathrm{ER}^{\mathrm{T} 2}$ occurred in our established $\mathrm{Tg}$ mice, we monitored spontaneous tumor progression, which might be caused by the activation of $\beta$-catenin in the absence of Tam. We found manifest thymic tumors specifically in Tg1 and Tg4 mice but not in the non-Tg littermates (Figure 2A and 2B). The tumor incidence in Tg1 mice increased from $23.6 \%$ ( 2 of 7 mice) at 10 months old to $63.7 \%$ (12 of 18 mice) at 12 months old (Figure 2A). Also, one of five Tg4 mice developed manifest thymomas at late onset (18 months) (Figure 2B). The low incidence of thymoma development in $\mathrm{Tg} 4$ mice might reflect the lower expression of $\Delta \mathrm{N} 64 \mathrm{Ctnnb} 1 / \mathrm{ER}^{\mathrm{T} 2}$ in the Tg4 thymus overall (Figure 1B). These data suggest that a leaky expression of activated $\beta$-catenin cause thymoma development in the $\mathrm{Tg} 1$ and $\mathrm{Tg} 4$ mice without Tam treatment.

We analyzed the thymoma phenotype in Tg1 mice and their non-Tg littermates at 2 months and 6 months of age. Although we could not observed manifest tumors in Tg1 thymi at 2 or 6 months, we observed microscopic thymoma lesions embedded in the CMJ of all Tg1 thymi $(n=4)$ at 2 months or formed an epithelial sheet in the medullary area of all Tg1 thymi $(n=7)$ at 6 months $(2$ and 6 months; Figure 2C). At 10 or 12 months, we found that the manifest thymic tumors of Tg1 mice were composed of polygonal epithelial cells with a squamoid appearance and scant lymphocytes (Figure 2C). Notably, perivascular spaces with loose connective tissues appeared between vessels and neoplastic epithelial sheets (10 and 12 months; Figure 2C), resembling the histology characteristics of type B3 thymoma, or atypical thymoma, in humans. Using immunofluorescence staining for K5 and K8, we showed that the early thymoma lesions expressed K5, but not K8 (2 months; Figure 2D). The K5-expressing lesions accumulated and formed a sheet-like pattern at 6 months that seemed to correspond to the pre-lesions of Tg1 thymomas at 10 and 12 months (Figure 2D). These $\mathrm{K} 5$-expressing lesions in $\mathrm{Tg} 1$ thymi at 6 months of age 
A

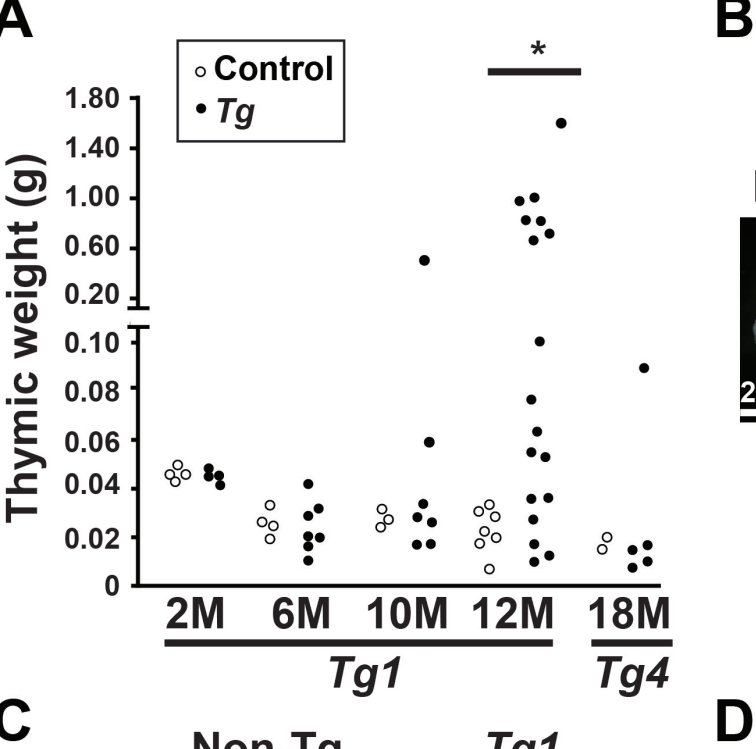

Non-Tg Tg1 (12M) Tg4 (18M)
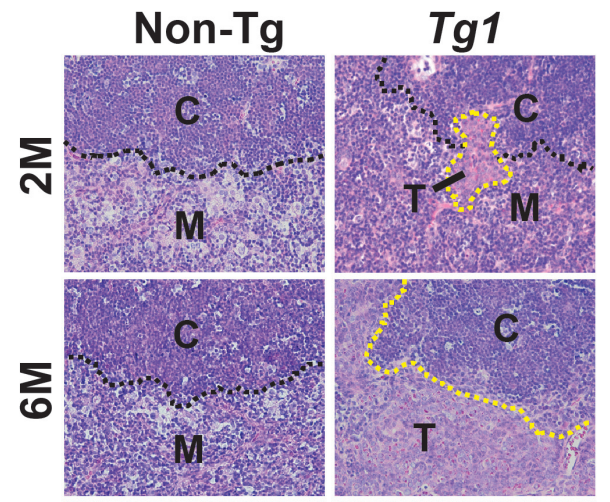

D
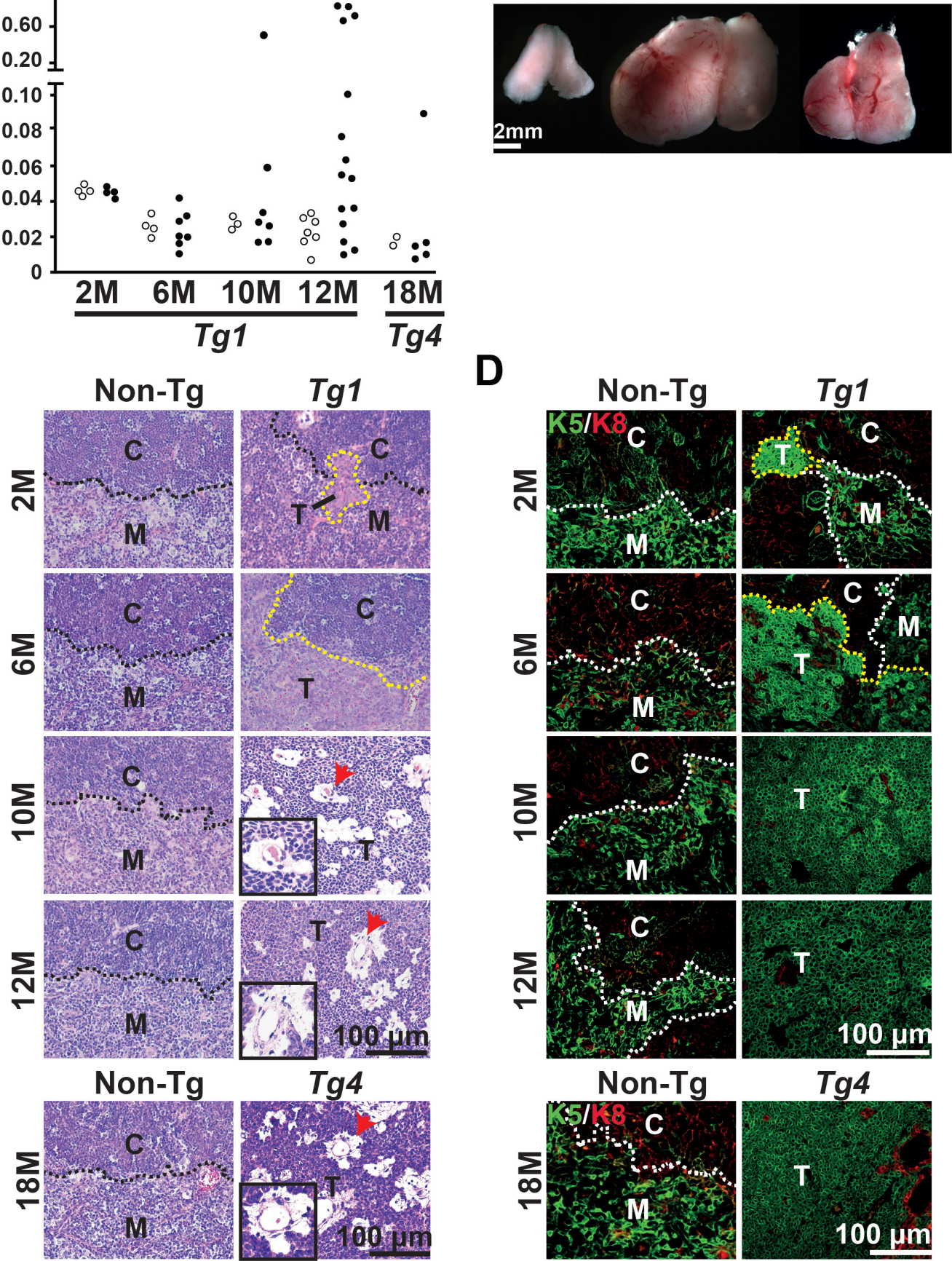

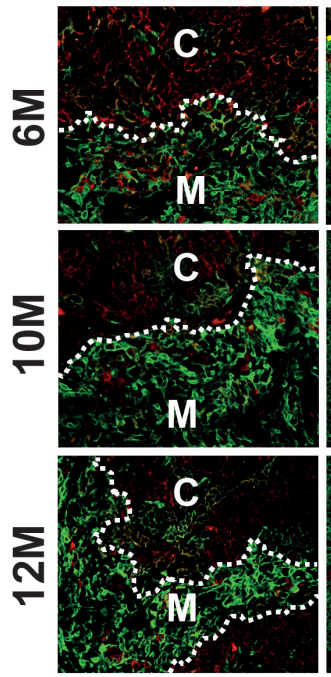

Non-Tg

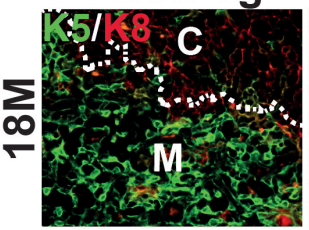

$100 \mathrm{um}$

$\operatorname{Tg} 4$

Figure 2: Spontaneous thymoma development in the Tg1 and Tg4 mice in the absence of tamoxifen (Tam) treatment. A. The thymi of non- $\operatorname{Tg}$ and $\operatorname{Tg} 1$ mice were weighed at 6, 8, 10, and 12 months of age. The thymi of Tg4 mice and non-Tg littermates were weighed at 18 months of age. Each circle represents an independent mouse. [*], $p<0.05$; B. Representative images of a non-Tg thymus, a spontaneous thymoma developed in one Tg1 mouse at 12 months old, and a spontaneous thymoma developed in one Tg4 mouse at 18 months are shown. C. H\&E staining of thymic sections reveals non-Tg thymi and thymoma development in Tg1 mice between 6 and 12 months. Lower panels are H\&E staining of a non-Tg thymus and a Tg4 thymoma at 18 months. C, cortex; M, medulla; T, tumor lesion; Black dashed lines, CMJ; Red arrows, perivascular space. Insets, high-powered view of perivascular space in thymomas; D. Coimmunofluorescent staining reveals the expression of K5 (green) and K8 (red) in the non-Tg thymi and thymoma lesions in the Tg1 (2, 6, 10, and 12 months) and Tg4 thymi (18 months). White dashed lines represent the CMJ. Yellow dashed lines depict the tumor boundary; Bar scale, $100 \mu \mathrm{m}$. 
were comparable to the features of short-term (3 days and 28 days) Tam-induced early lesions in the thymi of Tam-treated Tg1 mice (Figure 2D versus Figure 1F). Moreover, Tg4 tumors identified at 18 months exhibited similar histological features and K5 expression as those seen in Tg1 tumors at 10-12 months, indicating that lower transgenic $\beta$-catenin activation correlates with slower thymoma progression (Figure $2 \mathrm{C}$ and $2 \mathrm{D}$ ).

In addition, we performed flow cytometry to detect CD45 ${ }^{-} \mathrm{EpCAM}^{+}$gated UEA $1^{+}{ }^{+} \mathrm{Ly} 51^{-}$mTECs and UEA1Ly $51^{+}$cTECs in non-Tg thymi and Tg1 thymomas. In the non-Tg thymi, UEA1 ${ }^{+}$Ly $51^{-}$subset was reduced (approximately 2.5 -fold) and $\mathrm{UEA} 1^{+} \mathrm{Ly} 51^{+}$population was increased (approximately 3.5-fold) in 12-monthold mice compared to those subsets from 6-month-old mice (Supplementary Figure 4A and 4B), suggesting the changes of TEC subsets are associated with age. In the Tg1 thymi at 6 months old, all TEC subsets in CD45 $\mathrm{EpCAM}^{+}$cells were comparable to those in non-Tg thymi (Supplementary Figure 4A \& 4B). In the thymomas from 12-month-old Tg1 mice, we found that the collagenase could not completely dissociate the tumor fragments, resulting in a collagenase resistant portion (C-res) that could be subsequently dissociated by trypsin. Interestingly, the collagenase-dissociated and C-res subsets in the Tg1 thymomas were composed of higher percentage of

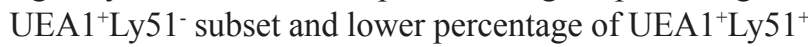
population compared to the TEC subsets of thymi from 12-month-old non-Tg mice (Supplementary Figure 4A \& 4B). Percentages of UEA $1{ }^{-} \mathrm{Ly} 51^{+}$subsets were comparable in non-Tg thymi and Tg1 thymoma (Supplementary Figure 4B). Therefore, it is possible that the sustained $\mathrm{K}^{+}$ and UEA $1^{+}$Ly $51^{-}$subsets in Tg1 thymomas arising from the mTECs, where the $\triangle \mathrm{N} 64 \mathrm{Ctnnb} 1 / \mathrm{ER}^{\mathrm{T} 2}$ transgene was expressed, maintained the differentiation characteristics of mTECs during thymoma progression.

\section{Activated Wnt/ $\beta$-catenin canonical pathway in the mouse thymomas}

To examine whether the Wnt/ $\beta$-catenin canonical pathway is activated in $\triangle \mathrm{N} 64 \mathrm{Ctnnb} 1 / \mathrm{ER}^{\mathrm{T} 2}$ thymomas in the absence of Tam, we introduced the TOP-Gal transgene to allow the measurement of $\mathrm{Wnt} / \beta$-catenin activation. Using whole-mount X-gal staining, we found that Tg1;TOP-Gal thymi clearly exhibited patched blue staining compared to negatively stained non-Tg littermate (TOP-Gal) thymi at 6 months (Figure 3A). Immunohistochemistry also revealed that the $\beta$-galactosidase was specifically detected in Tg1;TOP-Gal thymic medullae, but not in the non-Tg control (Figure 3B). We next examined several targeted genes of $\mathrm{Wnt} / \beta$-catenin canonical pathway, which should be activated if the $\Delta$ N64Ctnnb1/ER ${ }^{\mathrm{T} 2}$ transgene is functional during thymoma progression. Indeed, Axin2, Cyclin D1 and c-Myc were upregulated in the manifest thymomas compared to the CD45- thymic stromal cells isolated from non-Tg thymi (Figure 3C). Nuclear localization of $\beta$-catenin was also observed and significantly increased in the early lesions and manifest thymomas of Tg1 mice at 6 and 12 months of age (Figure $3 \mathrm{D}$ and $3 \mathrm{E})$. These data suggest that canonical $\mathrm{Wnt} / \beta$ catenin signaling is activated in thymomas and drives their progression.

\section{Compensation of endogenous $\beta$-catenin loss by $\triangle \mathrm{N} 64 \mathrm{Ctnnb1} / \mathbf{E R}^{\mathrm{T} 2}$ transgene for maintaining thymic homeostasis}

Previously, we have taken a loss-of-function approach to conditionally ablate $\beta$-catenin and demonstrate that $\beta$-catenin expression in TECs is intrinsically required for TEC differentiation, thereby affecting thymocyte development and resulting in thymic atrophy [6]. In this study, we examined whether Tg1 mice exhibited leaky $\beta$-catenin activity, which might be able to compensate for the conditional ablation of $\beta$-catenin in $\mathrm{K} 5$-expressing TECs and cause thymic atrophy. Thus, we bred the Tg1 mice to the previously established Ctnnb $1^{K 5-f x / f x}$ background to obtain $\operatorname{Tg} 1$; $C \operatorname{tnn} b 1^{K 5-f x / f x}$ mice. After 10 days of 4-OH-Tam (4-OHT) treatment, the size, cellularity, and CD4/CD8 distributions of $T g 1$; Ctnnb1 $1^{K 5-f x / f x}$ adult thymi were similar to those of thymi from control animals (Figure 4A-4C). These data suggest that the expression of constitutively active $\beta$-catenin in Tg1 mice is sufficient to rescue $C t n n b 1^{K 5-f x / f x}$ thymic atrophy. Furthermore, the $C t n n b 1^{K 5-f x / f x}$ thymic phenotype, including the decreased frequency of proliferating thymocytes (total, CD4-CD8$\mathrm{DN}$ and $\mathrm{CD}^{+} \mathrm{CD}^{+} \mathrm{DP}$; Figure 4D) and the increased percentage of annexin V-labeled apoptotic cells (total and $\mathrm{CD}^{+} \mathrm{CD}^{+} \mathrm{DP}$; Figure $4 \mathrm{E}$ ), was significantly reversed by the transgenic expression of stabilized $\beta$-catenin in K5expressing cells. Our data suggest that expression of the $\triangle \mathrm{N} 64 \mathrm{Ctnnb} 1 / \mathrm{ER}^{\mathrm{T} 2}$ fusion protein in TECs can functionally complement the effects of $\beta$-catenin loss in the K5expressing TEC lineage. This genetic complementation experiment also suggests that the $\Delta \mathrm{N} 64 \mathrm{Ctnnb} 1 / \mathrm{ER}^{\mathrm{T} 2}$ fusion protein in TECs can compensate endogenous $\beta$-catenin function.

\section{Aberrant expression of AIRE and p63 in mouse thymomas is similar to that in human thymomas}

The loss of AIRE [31] expression and intense p63 [32-34] expression have been reported in human thymomas and thymic carcinomas. Therefore, we determined the expression of AIRE and p63 in Tg1 thymoma samples and non-Tg thymi using co-immunofluorescence. We found that AIRE was undetectable in K5-expressing tumor cells of early thymoma lesions ( 2 or 6 months of age) or manifest thymomas of mice 10-12 months of age 

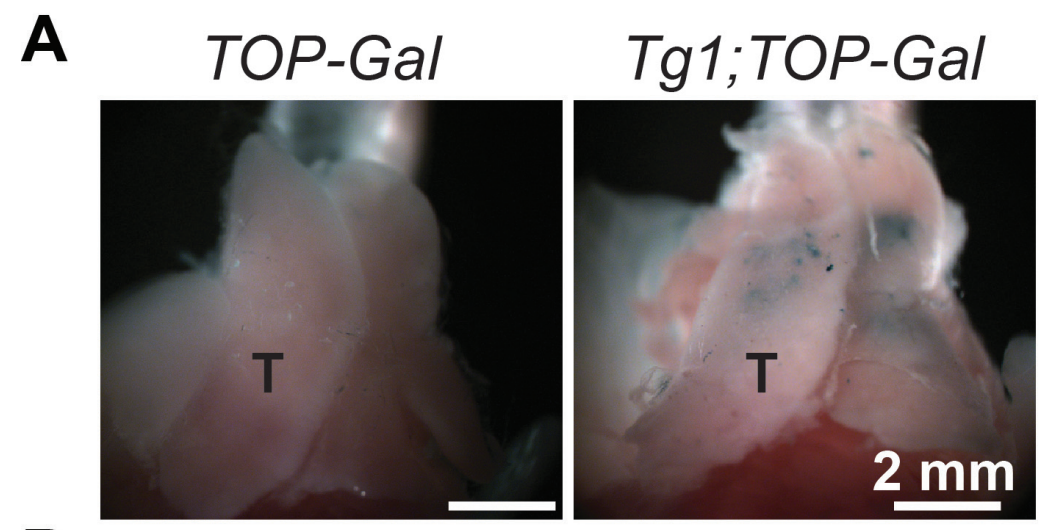

B

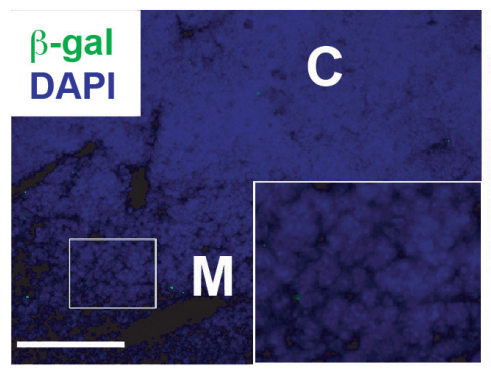

D
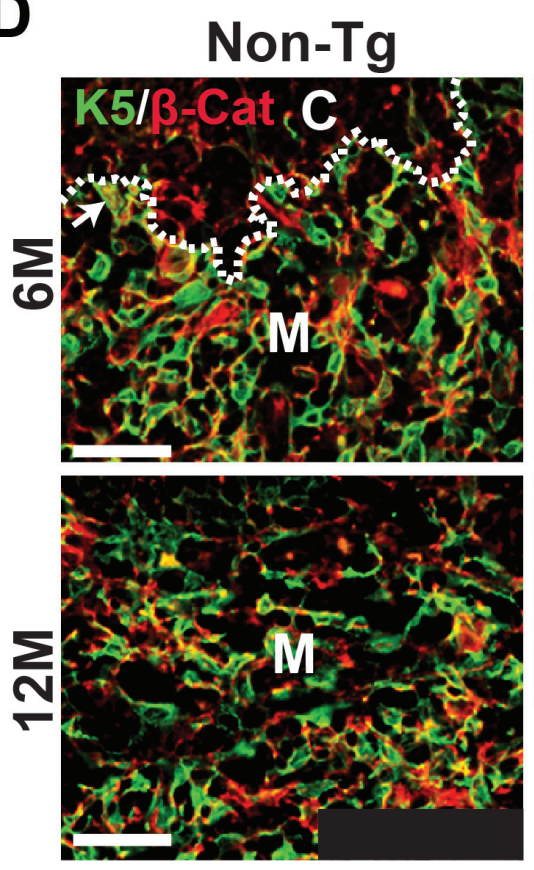

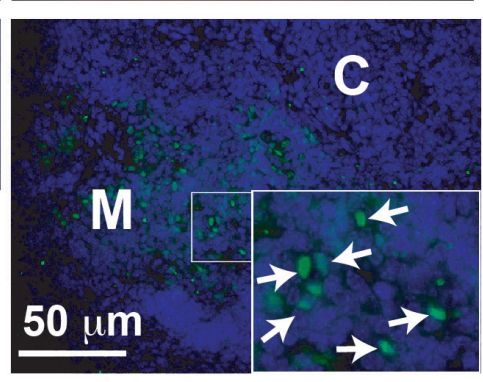

$\operatorname{Tg} 1$
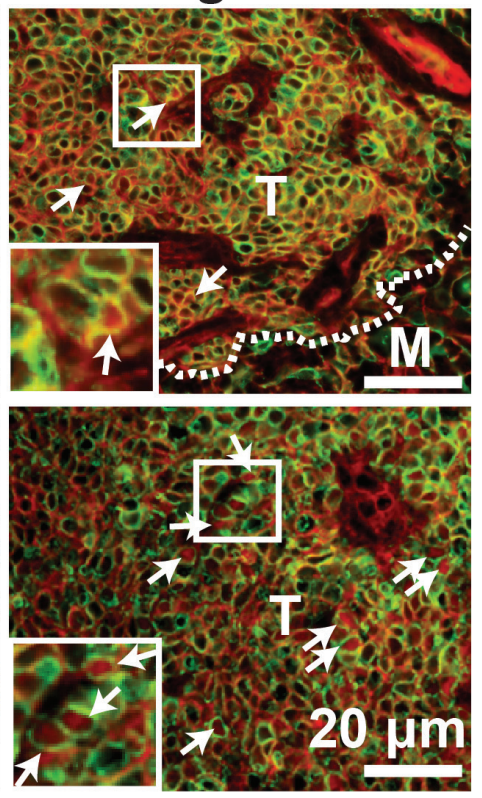

C

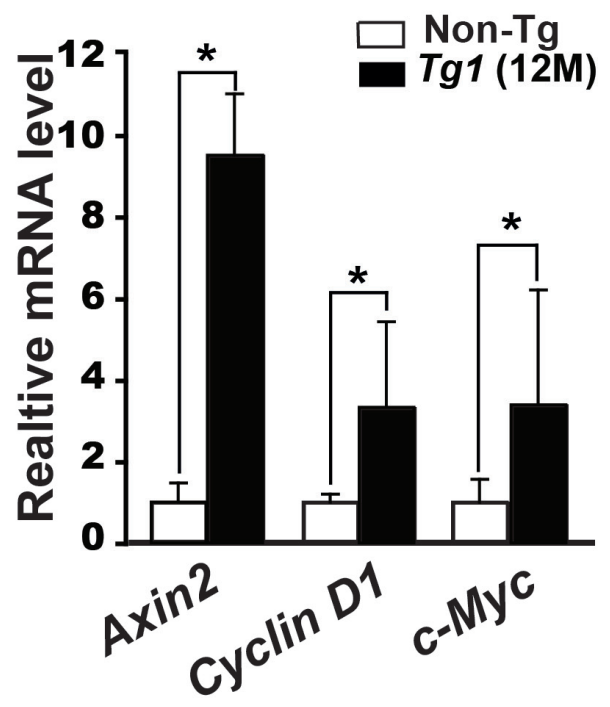

$\mathbf{E}$
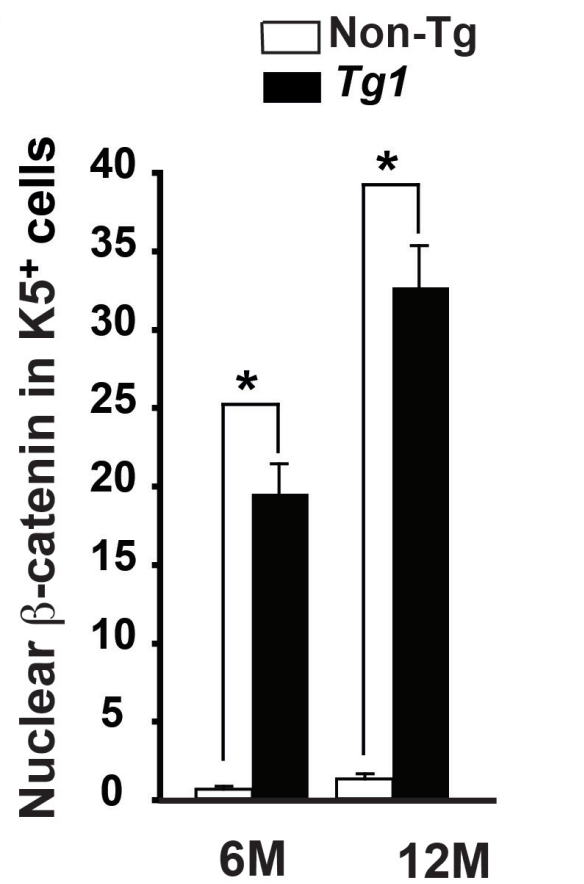

Figure 3: Activated Wnt/ $\beta$-catenin canonical pathway in the spontaneous Tg1 thymomas. A. $\beta$-galactosidase activity was determined by the whole-mount X-gal staining (blue) in the TOP-Gal and Tg1;TOP-Gal thymi ( $n=3$ each genotype) at 6 months. T, the thymus. B. Immunofluoresent staining of anti- $\beta$-galactosidase antibody reveals nuclear $\beta$-galactosidase expression (green) in Tg1; TOP$\mathrm{Gal}$, but not in the control (TOP-Gal) thymic medullae. Insets, high-power view of medulla; Arrows indicate nuclear $\beta$-galactosidase. DAPI (blue) was used for nuclear counterstaining. C. RT-qPCR reveals the relative expression levels of Wnt/ $\beta$-catenin canonical target genes, such as Axin 2, Cyclin D1 (Ccnd1), and c-Myc, in the CD45-EpCAM+ sorted TECs from 12-month-old control thymi and Tg1 thymomas $(n=3)$. [*], $p<0.05$. D. Co-immunofluorescent staining reveals the expression of $\mathrm{K} 5$ (green) and $\beta$-catenin (red) in the thymic sections of control and Tg1 mice at 6 and 12 months. White dashed lines, CMJ; Yellow dashed lines, the microscopic boundary of thymoma; Arrows, nuclear $\beta$-catenin; Insets, high-power view of thymoma lesions. E. Quantification of nuclear $\beta$-catenin in K5-expressing cells in non-Tg thymi and Tg1 thymomas at 6 and 12 months of age ( $n=3$, each group). [*], $p<0.05$. 
(Figure 5A). Comparatively, scattered AIRE expression was detected in medullary K5-expressing cells of the nonTg thymi at the corresponding time points (Figure 5A). In contrast, p63 showed intense homogenous expression in the K5-expressing tumor cells of both early thymoma lesions and manifest thymomas compared to the scattered p63 expression in K5-expressing cells of the non- Tg thymi (Figure 5B). Similar expression patterns of AIRE and p63 were also observed in a Tg4 thymoma at 18 months (Figure 5A and 5B). Interestingly, loss of AIRE and presence of p63 were observed in Tg1 thymomas with leaky $\beta$-catenin activation without Tam treatment, but these expression patterns were also found in a shortterm (3 days) treatment of Tam in Tg1 microscopic lesions (Supplementary Figure 5).

Next, we examined p63 transcripts in the manifest thymoma samples $(n=3)$ of Tg1 mice and purified CD45 thymic stromal cells of non-Tg mice $(n=3)$ using RT-qPCR. Notably, p63 can be transcribed from two alternative promoters, producing TAp63 and $\Delta \mathrm{Np} 63$ [35]. TAp63 and $\Delta \mathrm{Np} 63$ may each have three C-terminal variants, called $\alpha, \beta$ and $\gamma$, that are generated by alternative
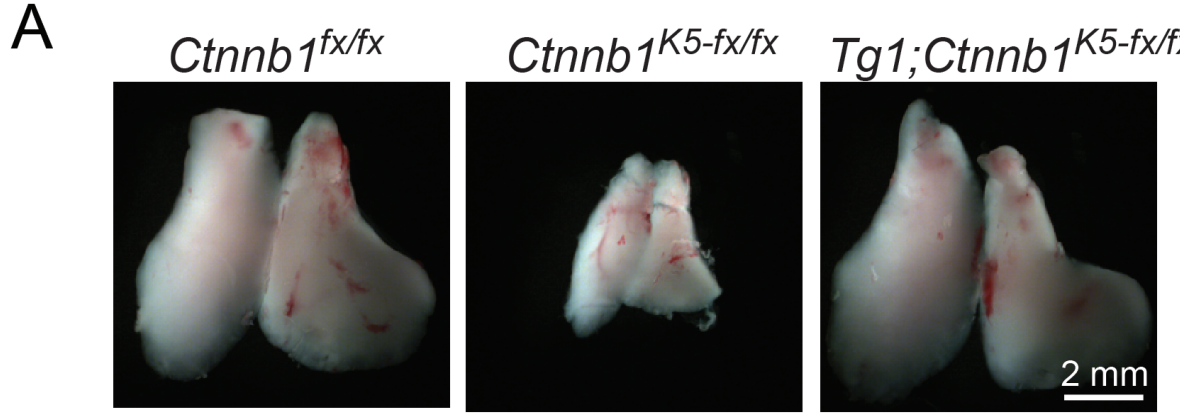

C

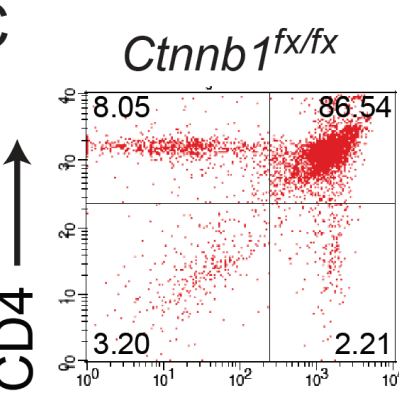

Ctnnb ${ }^{K} \mathrm{~K}-\mathrm{fx} / \mathrm{fx}$

$\operatorname{Tg} 1 ; \operatorname{Ctnn} b 1^{K 5-f x / f x}$
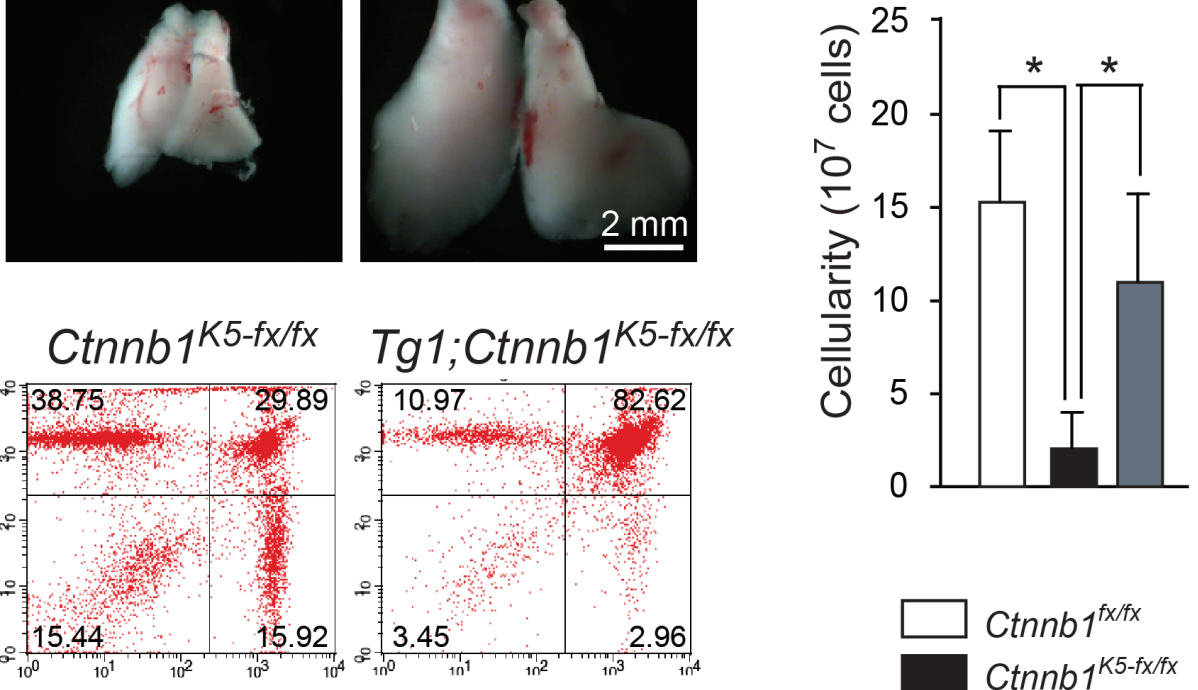

CD8

D

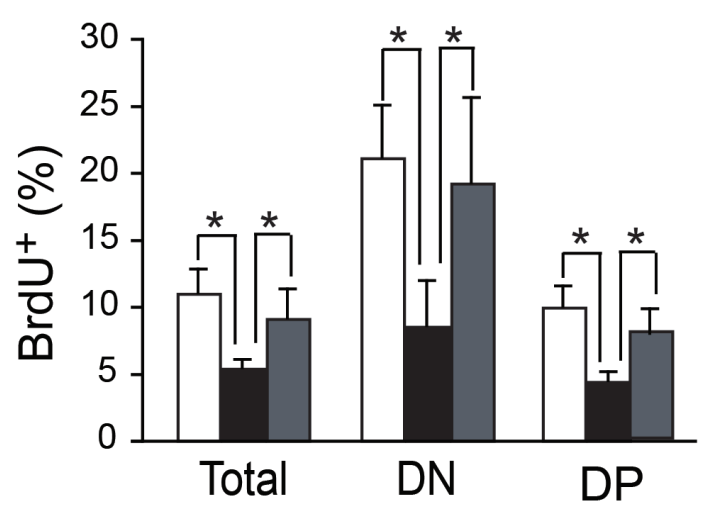

$\mathrm{E}$

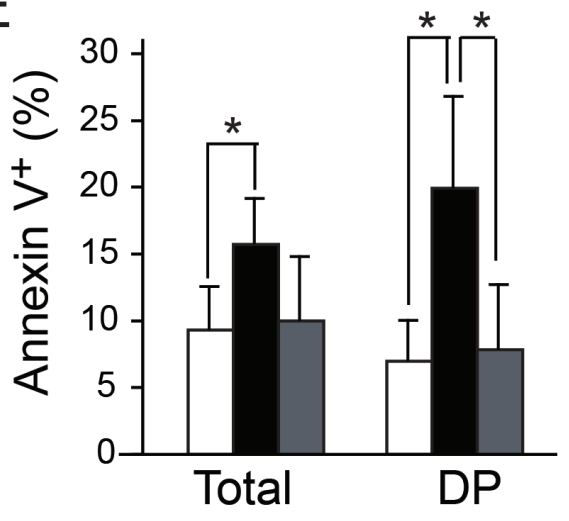

Figure 4: Expression of constitutively activated $\beta$-catenin in $\mathrm{K5}^{+}$lineage $\mathrm{TECs}$ rescues the thymopoiesis defects observed in Ctnnb1 ${ }^{\text {K5-fx/fx }}$ thymi. A. Gross view of the thymi of $C t n n b 1^{f x / f x}, C t n n b 1^{K 5-f x / f x}$ and $T g 1 ; C t n n b 1^{K 5-f x / f x}$ mice. The thymi of Tg1; Ctnnb $1^{K 5-f x / f x}$

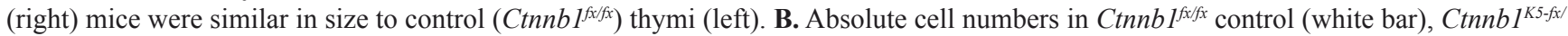
$f_{x}$ (black bar) and $T g 1 ; C \operatorname{tnnb} 1^{K s-f x / f x}$ thymi (gray bar) on day 10 post-4-OHT injection ( $n=4$ in each genotype). C. Representative dot plots show the similar CD4/CD8 expression profiles among developing thymocytes in $C t n n b 1^{f x / f x}, C t n n b 1^{K S-f x / f x}$ and $T g 1 ; C t n n b 1^{K S-f x / f x}$ thymi on day 10 post-4-OHT injection. D. Bar graphs represent the percentages of BrdU-labeled cells among total thymocytes, DN cells, and DP cells. E. Bar graphs represent the percentages of annexin V-labeled cells among total thymocytes and DP cells on day 10 post-4-OHT injection. $[*], p<0.05$. 
splicing of 3' exons [35]. Thus, we used isoform-specific primer sets to determine which p63 isoforms were expressed. Our results showed that $\Delta \mathrm{Np} 63$, but not TAp63, was increased in the manifest thymoma samples compared to the non-Tg thymic stromal cells (Figure 5C). Furthermore, the spicing variants $\Delta \mathrm{Np} 63-\alpha$ and $-\beta$, but not $-\gamma$, were upregulated in the manifest thymoma samples, suggesting that $\Delta \mathrm{Np} 63-\alpha$ and $-\beta$ were the major p63 isoforms involved with increased expression, which may contribute to tumorigenesis of TECs. Furthermore, one potential target gene of p53/p63 is p2 $1^{\mathrm{WAF} 1 / \mathrm{cip} 1}$, which can be suppressed by increasing levels of $\Delta \mathrm{Np} 63$ isoforms [36-39]. We found that p21 transcript was significantly decreased in Tg1 thymomas compared to non- $\mathrm{Tg}$ CD45- thymic stromal cells (Figure 5C), a finding that is consistent with p21 downregulation in human thymomas [40].

\section{Aberrant expression of $\beta 5 t$ and squamoid differentiation markers in the mouse thymomas}

$\beta 5 \mathrm{t}$ is a cTEC-specific proteosomal subunit [41, 42] that has recently been used as a differential diagnosis marker of type B3 thymomas [43, 44]. To gain insight into the phenotypes of mouse thymomas developed in Tg1 mice, we examined the expression of $\beta 5 \mathrm{t}$ in $\mathrm{Tg} 1$ thymomas during initiation and progression. Using immunohistochemistry, we detected $\beta 5 \mathrm{t}$ in the cortical area in control thymi of Tg1 mice aged 2 to 12 months (Figure 6A). In 2-month-old Tg1 thymi, $\beta 5 \mathrm{t}$ was expressed in the cortical area similarly to non-Tg thymi and was weakly detected in focal microscopic lesions in medullary area (Figure 6A). As thymoma development proceeded, robust $\beta 5 \mathrm{t}$ expression was detected in most neoplastic cells from thymomas of 6- to 12-month-old Tg1 mice (Figure 6A). These findings suggest that $\beta 5 \mathrm{t}$ expression increased with age in Tg1 thymomas. This characteristic resembles that of type B3 thymomas in humans [43, 44]. Furthermore, we examined $\beta 5 \mathrm{t}$ and $\beta$-catenin expression in the early lesions in the thymi of Tg1 mice at 6 months of age and in manifest thymomas of Tg1 mice at 10-12 months of age. We found that the nuclear form of $\beta$-catenin and uniform expression of $\beta 5 \mathrm{t}$ were markedly increased in manifest at 12-month-old thymomas compared with that of the early lesions at 6 months of age (Figure 6B). Thus, transgenic mice expressing stabilized $\beta$-catenin in TECs exhibit thymoma development and molecular characteristics, such as $\beta 5 \mathrm{t}$, that resemble that of type B3 thymomas in humans.

While previous studies showed that Apc loss or $\beta$-catenin stabilization in TECs caused squamous metaplasia in the developing thymus $[26,28]$, no thymoma phenotype was reported. To characterize TECs-derived thymomas in $\operatorname{Tg} 1$ mice, the expression of squamoid differentiation markers $\mathrm{K} 10$ and loricrin $[45,46]$ were used in immunohistochemistry. We found focal positive for K10 expression (Figure 6C) and scattered positive for loricrin expression (Figure 6D) in manifest thymomas from 12-month-old Tg1 mice. The heterogenously expressed K10 and loricrin indicated aberrant squamoid cell-fate differentiation in some neoplastic cells of Tg1 thymomas.

\section{DISCUSSION}

In this study, the transgenic mice expressing $\triangle N 64 C t n n b 1 / E R^{T 2}$ under the control of $\mathrm{K} 5$ promoter enable us to monitor thymoma progression from early lesions to manifest thymomas. These thymomas have histological and molecular characteristics that resemble those of type B3 thymomas in humans. Our findings also suggest that induced $\beta$-catenin activation is sufficient to drive thymoma initiation, forming microscopic lesions. Loss of AIRE expression and gain of $\Delta \mathrm{Np} 63-\alpha$ and $-\beta$ isoform expression in the microscopic lesions, as well as in the manifest thymomas, appear to be common features between this mouse model and human thymomas [31-34]. Interestingly, thymomas developed in the $\mathrm{Tg} 1$ mice also express $\beta 5 \mathrm{t}$, which is a differential diagnostic marker for type B3 thymomas in humans $[43,44]$. Both K10 and loricrin were focally expressed in some neoplastic cells in Tg1 thymomas, suggesting that over-expression of the $\triangle \mathrm{N} 64 \mathrm{Ctnnb} 1 / \mathrm{ER}^{\mathrm{T} 2}$ fusion protein in TECs may adopt squamous cell-fate differentiation.

Although the molecular genetics of human thymomas are largely unclear, the presence of $A P C$ mutations has been linked to pathogenesis of type B3 thymomas [47]. Also, $\mathrm{LOH}$ at $A P C$ locus has been found in the type B thymomas $[24,25]$. These reports implicate the possible link of APC-controlled $\beta$-catenin activation in human thymomas. Previously, Apc conditional ablation by K14-Cre mice showed aberrant growth of epidermalderived organs and squamous metaplasia in the thymus [26]. However, these Apc-conditional knock-out mice (K14-Cre; $A p c^{C K O / C K O}$ ) die before weaning, thus precluding follow-up studies to monitor thymic homeostasis and thymoma development. In our study, the transgenic mice expressing the $\Delta \mathrm{N} 64 \mathrm{Ctnnb} 1 / \mathrm{ER}^{\mathrm{T} 2}$ fusion protein exhibited leaky $\beta$-catenin activation in $\mathrm{K} 5$-expressing cells, but this phenotype did not lead to early lethality, thus enabling us to monitor thymoma development as the transgenic animals aged. Induction with tamoxifen initiated thymoma lesions in a temporally control manner, enabling us to examine molecular changes in the early stages of thymoma progression. The thymomas that developed in the Tg1 and $\mathrm{Tg} 4$ mice reflect the expression levels of the $\Delta$ N64Ctnnb1/ $\mathrm{ER}^{\mathrm{T} 2}$ transgene and share histological appearance (e.g. cellular atypia and perivascular space) and expression patterns of cellular markers (AIRE, p63, p21 and $\beta 5 \mathrm{t}$ ) with those of human thymomas. Therefore, these findings suggest that the $\beta$-catenin activation is possible to drive thymoma pathogenesis. 
A
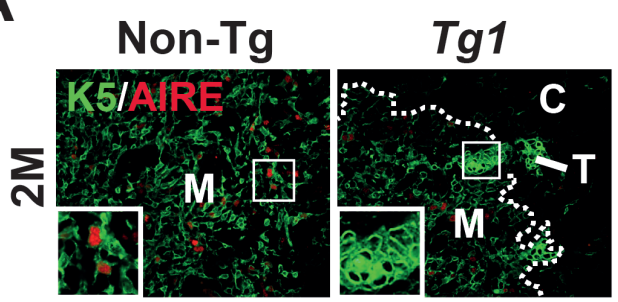

B
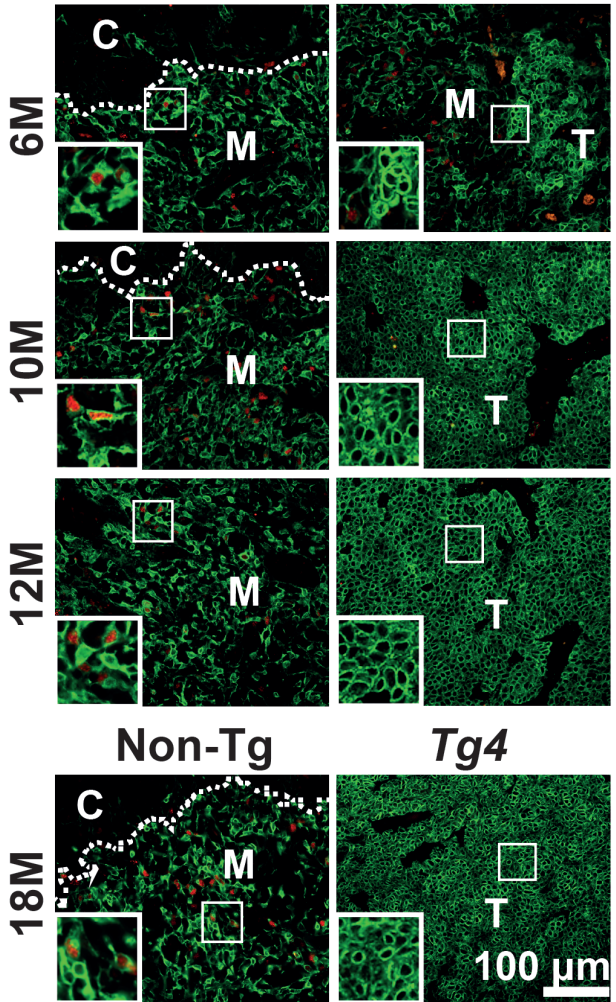

$\operatorname{Tg} 4$

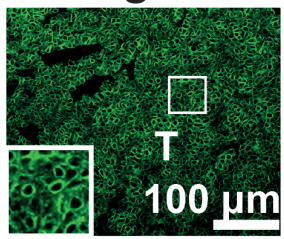

C
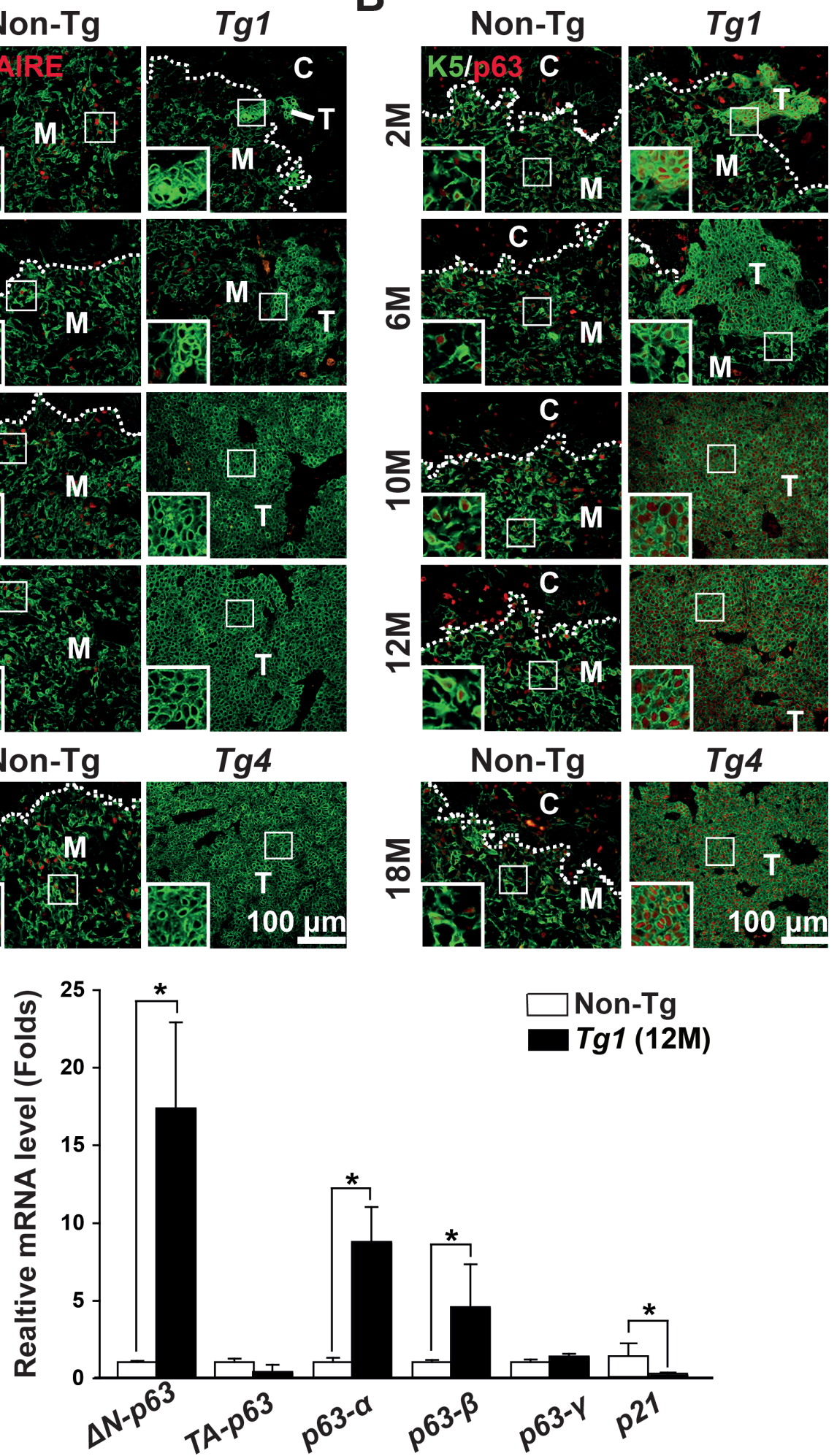

$\operatorname{Tg} 4$

Figure 5: AIRE, p63 isoforms, and p21 waf1/cip expression in mouse thymomas. A. Co-immunostaining of K5 (green) and AIRE (red) reveals scattered expression of AIRE in the K5-expressing cells of the non-Tg thymi, but loss of AIRE in the thymoma lesions of the $\mathrm{Tg} 1$ and $\mathrm{Tg} 4$ mice at the indicated time points. B. Co-immunostaining of K5 (green) and p63 (red) reveals scattered expression of p63 in the K5-expressing cells of the non-Tg thymi and uniformed expression of p63 in the thymoma lesions of the Tg1 and Tg4 mice at the indicated time points. Insects, high-power view of boxed areas; C. Bar graphs represent relative expression levels of p63 isoforms (TAp63, $\Delta \mathrm{Np63}$, p63- $\alpha$, p63- $\beta$ and p63- $\gamma$ ) and the p53/p63 target gene p21 wafl/cip in CD45- thymic stromal cells of non- $\operatorname{~g~thymi~and~in~Tg1~thymomas~}(n=$ 3 in each group) at 12 months using RT-qPCR analysis. [*], $p<0.05$. 
Genetically engineered mouse models are extremely valuable for the modeling of human diseases because they enable us to validate causative mediators using both loss- and gain-of-function approaches. Diseases such as thymoma, which have various clinically unsolved problems, are difficult to tackle because of the rarity of clinical cases reported. Previously, SV40 T-antigen, driven by its own viral early region elements, which include an enhancer and a promoter, caused thymic epithelial carcinomas in transgenic mice [48]. Additionally, forced expression of the E6/E7 oncogene driven by the K5 promoter caused thymic epithelial tumors in the transgenic mice [49]. These viral oncogenes are commonly used for transgenic studies to block p53 or Rb checkpoints in the cell cycle, leading to tumorigenesis [50]. In addition, overexpression of E2F2, which is a E2F member and downstream target of $\mathrm{Rb}$, alters cell cycle progression [51] in transgenic mice, causing a high incidence of thymomas [52]. These transgenic mouse models suggest that dysregulated cell cycle control can be the critical determinant for thymoma development. Consistently, the levels of cell cycle regulators p21, p27, and p53 have been examined in encapsulated thymomas in a clinical study [40]. Increased p53 and decreased p21 and p27 in thymoma tissue correlated with poor prognosis for shorter disease-free survival [40], suggesting that aberrant regulation of cell-cycle progression contributes to the clinical outcomes of the patients with thymomas. In this study, we also demonstrated downregulated p21, which was coincidently associated with upregulated $\Delta \mathrm{Np} 63-\alpha$ and $-\beta$ isoforms, which may work together to promote TECs tumorigenesis.

In summary, our study provides data to generate a fundamental basis for understanding the activation of $\beta$-catenin in the progression of thymic epithelial carcinogenesis. In the future, our mouse models may be used as the in vivo platforms for validating the therapeutic agents and strategies to treat the thymomas.
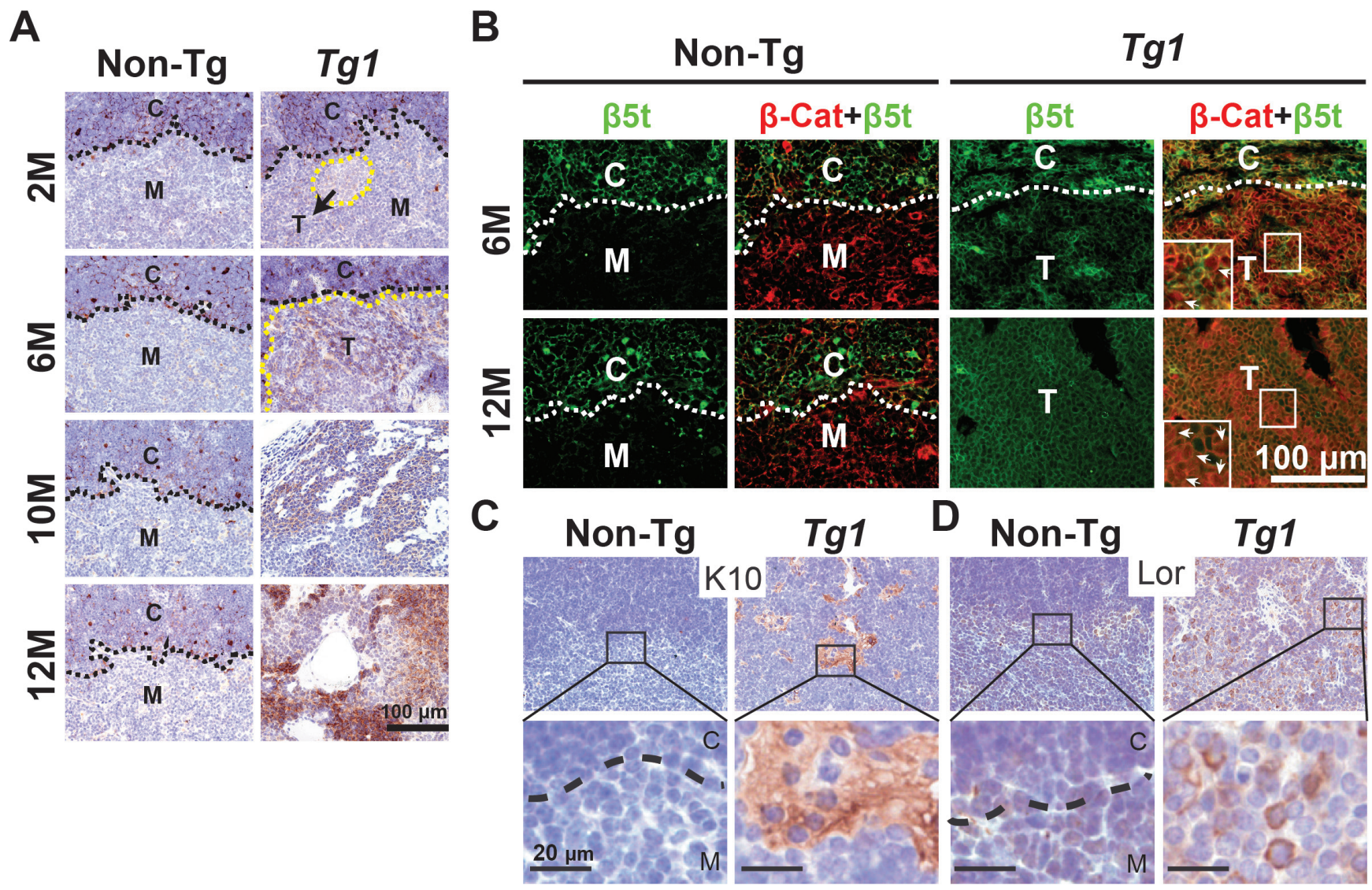

Figure 6: Aberrant $\beta 5$ t and squamoid differentiation markers expression in Tg1 mouse thymomas. A. Immunohistochemistry reveals intense $\beta 5 \mathrm{t}$ expression (brown) in the cortex, but not in the medulla, of non- $\mathrm{Tg}$ thymi, and in the $\mathrm{Tg} 1$ thymoma lesions arising in the CMJ and medulla at 2, 6, 10, and 12 months. Hematoxylin is used for counterstaining. Black dashed lines represent the CMJ. Yellow dashed lines depict the thymoma boundary. B. Immunofluorescent staining of $\beta 5 \mathrm{t}$ (green) reveals $\beta 5 \mathrm{t}$ expression in cTECs of non-Tg thymi and in the Tg1 thymoma lesions at 6 and 12 months. Merged images of $\beta 5 \mathrm{t}$ (green) and $\beta$-catenin (red) co-immunostaining reveal that both proteins are co-expressed in the thymomas. Insets: high-power views of boxed areas that show stabilized $\beta$-catenin in both the cytoplasm and the nuclei (arrows); White dashed lines, CMJ; C, cortex; M, medulla; T, thymoma lesions. C. and D. Immunohistochemistry using anti-K10 and loricrin antibodies reveals focal K10 and scattered loricrin (lor) expression (brown) in the Tg1 thymomas compared to weak staining in the non-Tg thymi. Lower panels: high-power views of boxed areas in upper panels. 


\section{MATERIALS AND METHODS}

\section{Mice and genotyping}

$T g\left(K 5-\triangle N 64 C t n n b 1 / E R^{T 2}\right)$ transgenic mouse lines $\mathrm{Tg} 1, \mathrm{Tg} 4$, and $\mathrm{Tg} 10$ were generated on the $\mathrm{C} 57 \mathrm{BL} / 6$ background for this study. The K5-4N64Ctnnb1/ER $R^{T 2}$ transgenic plasmid (Figure 1A) was composed of the bovine keratin 5 promoter (derived from BK5-Cre plasmid, which was kindly provided by Dr. José L. Jorcano), a rabbit $\beta$-globulin intron sequence (IVS), a Myc-tagged sequence, two copies of a nuclear location signal (NLS), a portion of the Ctnnbl encoding N-terminal 64-amino acid deletion mutant of $\beta$-catenin, the mutated ligand-binding domain of human estrogen receptor $\left(E R^{T 2}\right)$ gene (derived from Cre-ER ${ }^{\mathrm{T} 2}$ plasmid, which was kindly provided by Dr. Pierre Chambon, IGBMC, France), an SV40 polyadenylation signal (pA), and two copies of the HS4 insulators. The 11.4-kb NotI/SalI-digested transgene fragment was eluted and separated from the pBluescript vector backbone for pronuclear microinjection. TOPGal transgenic mice [53] were obtained from the RIKEN biological resource center (Experimental Animal Division, RIKEN BRC, Ibaraki, Japan), and then bred with Tg1 to generate Tg1;TOP-Gal mice. Ctnnb $1^{K S-f x}$ ${ }^{f x}$ mice carrying the $B K 5-C r e E R^{T}$ transgene [54] and the Ctnnb1 conditional allele [55] were described previously [6]. Tg1;Ctnnb $1^{K 5-f x / f x}$ mice were generated by crossing Ctnnb $1^{K S-f x /+}$ with Tg1;Ctnnb $1^{f x /+}$ mice. Allelic specific genotyping was conducted by PCR methodology. The $T g\left(K 5-\triangle N 64 C\right.$ tnnb $\left.1 / E R^{T 2}\right)$ transgene was detected using the Myc-tag forward primer (5'-gct cat ttc tga aga gga ctt g-3') and Ctnnb1 reverse primer (5'-gct cag gaa ttg cac gtg tgg-3'), yielding a 400-bp PCR product. The TOP-Gal transgene was detected with forward primer ( $5^{\prime}-\mathrm{ttg} \mathrm{ccg}$ tct gaa $\mathrm{ttt}$ gac ctg-3') and reverse primer (5'-tct gct tca atc agc gtg cc-3'), yielding a 500-bp PCR product. Genotyping of $C t n n b 1^{K S-f x / f x}$ mice was described previously [6]. All experiments with mice were performed with the approval of the Institutional Animal Care and Use Committee (IACUC) at National Yang-Ming University.

\section{Tamoxifen administration}

Tamoxifen (Tam) (Sigma-Aldrich, St. Louis, MO, USA) was dissolved in $100 \%$ ethanol $(10 \mathrm{mg} / \mathrm{ml})$ and then mixed in sunflower seed oil (oil; Sigma-Aldrich) before intraperitoneal injection. Mice at 8 weeks of age were injected with Tam $(0.5 \mathrm{mg}$ in $100 \mu \mathrm{l}$ oil $)$ or vehicle (oil) only for 5 consecutive days. 4-OH-Tam (4-OHT) was dissolved in DMSO and then emulsified in oil as described previously $[6,54,56]$. Mice at the 8 weeks of age were injected intraperitoneally with three doses of 4-OHT $(0.1$ $\mathrm{mg}$ in $250 \mu \mathrm{l}$ oil) every other day.

\section{Western blot assay}

Generally, the experimental procedures of western blot assay were completed as described previously [56]. Primary antibodies against $\beta$-catenin (mouse IgG. 1:1000; BD Bioscience) and GAPDH (mouse IgG, 1:2000; Santa Cruz) were used for these analyses.

\section{Flow cytometry and sorting of TECs}

Experimental procedure was performed as described previously [6]. Briefly, the thymic fragments dissected from non-Tg and Tg mice were treated with collagenase (1 $\mathrm{mg} / \mathrm{mL}$ collagenase D; Sigma $)$ and DNase I $(0.1 \mathrm{mg} / \mathrm{mL}$; Sigma) at $37^{\circ} \mathrm{C}$ for 15 minutes. Subsequently, collagenase $\mathrm{D}$ activity was terminated by adding phosphate buffered saline (PBS) containing 1\% FBS and 0.5 mM EDTA. In addition, the portion of $\mathrm{Tg} 1$ thymomas that could not be completely dissociated by collagenase was collected and subsequently treated with Trypsin $(0.05 \%)$-EDTA $(0.02 \%)$ (Invitrogen) for preparing the single cell suspension. Finally, the cell suspension were washed with PBS and then stained with APC-congujated anti-CD45 antibody (1:200; Biolegend, San Diego, CA, USA), PE/Cy7conjugated anti-EpCAM antibody (1:400; eBiocience, San Diego, CA, USA), Rhodamine-conjugated UEA1 (1:50; Vector Laboratories, Burlingame, CA, USA) and FITCconjugated anti-Ly51 antibody (1:400; Biolegend) for the following flow cytometry analysis by BD FACScalibur (BD Biosciences). For cell sorting experiment, the UEA $1^{+}$ and $\mathrm{Ly} 51^{+}$subsets were gated and sorted from CD45 $\mathrm{EpCAM}^{+} \mathrm{TEC}$ populations by a BD FACSAria cell sorter (BD Biosciences).

\section{Quantitative RT-PCR (RT-qPCR)}

Detailed experimental procedures were described previously [6]. Expression of $\triangle N 64 C t n n b 1 / E R^{T 2}$ was detected by the transgene-specific Myc-NLS primers (5'GAG CAAAAG CTC ATT TCT GAA-3' and 5'-TAC TTG CTC TTG CGT GAA GG-3') or ER ${ }^{\mathrm{T} 2}$ primers (5'-CTT GCT CTT GGA CAG GAA CC-3' and 5'-CGA GAT GAT GTA GCC AGC AG-3'). Expression of K5 was detected using primers 5'- ACA GGA AGC TGC TGG AGG GC3' and 5'-GGT GGA GAC AAA TTT GAC ACT GG-3'. Expression of Aire was detected using primers 5'-GGT TCC TCC CCT TCC ATC-3' and 5'-GGC ACA CTC ATC CTC GTT CT- 3 '. Expression of $\beta 5 t$ was detected by primers 5'-GCT GTA TAG GGA GCT GCA GAA -3' and 5'-TGG CAG GCT CAG GAT AGA TT-3'. Expression of Axin2 was detected using primers 5'-ctg ctg gtc agg cag gag-3' and 5'-tgc cag ttt ctt tgg ctc tt-3'. Expression of Ccnd1 was determined using the primers 5 '-ctg gcc atg aac tac ctg ga-3' and 5'-gtc aca ctt gat cac tct gg-3'. 
Expression of c-Myc was determined using the primers 5'-cga aac tct ggt gca taa act g-3' and 5' -gaa ccg ttc tcc tta get ctc a-3'. Expression $\Delta N$ p63 and TAp63 isoforms was detected using a common reverse primer 5' -gag gag ccg ttc tga atc tg-3' with the forward primers 5'-caa aac cet gga agc aga aa-3' or 5'-gtg gat gaa cct tcc gaa aa-3', respectively. The alternatively spliced p63- $\alpha$ isoform was detected using primers 5'-cac tct cca tgc cet cca-3' and 5 '-gcc caa cet tgc taa gaa act -3 '. The other alternative splice variants, $p 63-\beta$ and $-\gamma$, were detected using common forward primer 5'-tcc ctc agc aca cga tcg a-3' and specific reverse primers 5' -act tgc caa atc ctg aca atg c-3' and 5'gac gtc aga ctg tgt cgg agc-3', respectively. Expression of $p 21^{\text {WAFI/CIP1 }}$ was detected using the primers 5'-ttg cca gca gaa taa aag gtg-3' and 5'-ttt get cct gtg cgg aac-3'. Ribosomal RNA L19 was used as internal control and detected with the primers 5'-tcg ttg ccg gaa aaa cac-3' and 5'-agg tca cct tct cag gca tc-3'.

\section{Characterization of thymoma phenotypes}

Hematoxylin \& eosin (H\&E) staining, immunofluorescence and immunohistochemistry were conducted to characterize the thymomas developed in the Tg1 and Tg4 mice. The detail experimental procedures for the tumor phenotype characterization have been described in our previous studies [6, 56-58]. The primary antibodies against K5, K8, $\beta$-catenin, AIRE, and p63 were described previously [6]. Anti- $\beta 5$ t antibody (rabbit IgG, Medical and Biology Laboratories, Nagoya, Japan) was diluted at 1:400 for both immunohistochemistry and immunofluorescence staining in this study. Anti-K10 (rabbit IgG, Abcam, Cambridge, UK) and anti-loricrin (rabbit IgG, Zymed Laboratories, South San Francisco, USA) were diluted at 1:200 and 1:1000, respectively, for immunohistochemistry.

\section{Expression and activation of TOP-Gal}

The experimental procedures of whole-mount X-gal staining were described previously [54, 58, 59]. The antibody against $\beta$-galactosidase (rabbit IgG, 1:1000; MP Biomedicals, Santa Ana, California, USA) used in immunostaining was performed as described previously [60].

\section{Flow cytometry analysis of thymocytes}

CD4/CD8 profiles, BrdU labeling, and Annexin V-binding of thymocytes were conducted by flow cytometry analysis as described in a previous study [6].

\section{Statistical analysis}

All statistical data were analyzed using the paired Student's t-test for statistical comparisons. $P<0.05$ was defined as statistically significant.

\section{ACKNOWLEDGMENTS}

The authors thank Yu-Lei Chang for initial assistance. This work was supported by a grant from the National Health Research Institutes (grant number NHRIEX104-10240BI), a grant from the Ministry of Education "Aim for the Top University Plan" and grants from the Ministry of Science and Technology to C.-M. C. (grant numbers NSC 102-2325-B-010-015, MOST 103-2325B-010-002 and MOST 103-2320-B010-030) and to the Taiwan Mouse Clinic (grant number MOST 103-2325-B001-015).

\section{Author contributions}

Designed the experiments, analyzed the data and wrote the paper: C.-C.L., L.-R.Y. and C.-M.C.; Performed the experiments: C.-C.L., T.-L.L. and Y.-R. Y.

\section{CONFLICTS OF INTEREST}

The authors have no conflicting financial interests.

\section{REFERENCES}

1. Anderson $G$ and Takahama Y. Thymic epithelial cells: working class heroes for $\mathrm{T}$ cell development and repertoire selection. Trends Immunol. 2012; 33:256-263.

2. Shezen E, Okon E, Ben-Hur H and Abramsky O. Cytokeratin expression in human thymus: immunohistochemical mapping. Cell Tissue Res. 1995; 279:221-231.

3. Klug DB, Carter C, Crouch E, Roop D, Conti CJ and Richie ER. Interdependence of cortical thymic epithelial cell differentiation and T-lineage commitment. Proc Natl Acad Sci USA. 1998; 95:11822-11827.

4. Gill J, Malin M, Hollander GA and Boyd R. Generation of a complete thymic microenvironment by MTS24(+) thymic epithelial cells. Nat Immunol. 2002; 3:635-642.

5. Lomada D, Liu B, Coghlan L, Hu Y and Richie ER. Thymus Medulla Formation and Central Tolerance Are Restored in IKK $\alpha-/-$ Mice That Express an IKK $\alpha$ Transgene in Keratin 5+ Thymic Epithelial Cells. J Immunol. 2007; 178:829-837.

6. Liang C-C, You L-R, Yen JJ-Y, Liao N-S, Yang-Yen H-F and Chen C-M. Thymic epithelial beta-catenin is required for adult thymic homeostasis and function. Immunol Cell Biol. 2013; 91:511-523. 
7. Dooley J, Erickson M and Farr AG. Alterations of the Medullary Epithelial Compartment in the Aire-Deficient Thymus: Implications for Programs of Thymic Epithelial Differentiation. J Immunol. 2008; 181:5225-5232.

8. Heino M, Peterson P, Sillanpää N, Guérin S, Wu L, Anderson G, Scott HS, Antonarakis SE, Kudoh J, Shimizu $\mathrm{N}$, Jenkinson EJ, Naquet $\mathrm{P}$ and Krohn KJE. RNA and protein expression of the murine autoimmune regulator gene (Aire) in normal, RelB-deficient and in NOD mouse. Eur J Immunol. 2000; 30:1884-1893.

9. Heino M, Peterson P, Kudoh J, Nagamine K, Lagerstedt A, Ovod V, Ranki A, Rantala I, Nieminen M, Tuukkanen J, Scott HS, Antonarakis SE, Shimizu N and Krohn K. Autoimmune Regulator Is Expressed in the Cells Regulating Immune Tolerance in Thymus Medulla. Biochem Biophys Res Commun. 1999; 257:821-825.

10. Nishikawa Y, Hirota F, Yano M, Kitajima H, Miyazaki J-i, Kawamoto H, Mouri Y and Matsumoto M. Biphasic Aire expression in early embryos and in medullary thymic epithelial cells before end-stage terminal differentiation. $\mathrm{J}$ Exp Med. 2010; 207:963-971.

11. Fletcher AL, Lowen TE, Sakkal S, Reiseger JJ, Hammett MV, Seach N, Scott HS, Boyd RL and Chidgey AP. Ablation and Regeneration of Tolerance-Inducing Medullary Thymic Epithelial Cells after Cyclosporine, Cyclophosphamide, and Dexamethasone Treatment. J Immunol. 2009; 183:823-831.

12. Jenkinson WE, Nakamura K, White AJ, Jenkinson EJ and Anderson G. Normal T cell selection occurs in CD205deficient thymic microenvironments. PLoS ONE. 2012; 7:e53416.

13. Hamazaki Y, Fujita H, Kobayashi T, Choi Y, Scott HS, Matsumoto $\mathrm{M}$ and Minato N. Medullary thymic epithelial cells expressing Aire represent a unique lineage derived from cells expressing claudin. Nat Immunol. 2007; 8:304311.

14. Yang SJ, Ahn S, Park CS, Holmes KL, Westrup J, Chang $\mathrm{CH}$ and Kim MG. The quantitative assessment of MHC II on thymic epithelium: implications in cortical thymocyte development. Int Immunol. 2006; 18:729-739.

15. Akiyama T, Maeda S, Yamane S, Ogino K, Kasai M, Kajiura F, Matsumoto M and Inoue J-i. Dependence of SelfTolerance on TRAF6-Directed Development of Thymic Stroma. Science 2005; 308:248-251.

16. Ripen AM, Nitta T, Murata S, Tanaka K and Takahama Y. Ontogeny of thymic cortical epithelial cells expressing the thymoproteasome subunit $\beta 5$ t. Eur J Immunol. 2011; 41:1278-1287.

17. Murata S, Sasaki K, Kishimoto T, Niwa S-i, Hayashi H, Takahama Y and Tanaka K. Regulation of CD8+ T Cell Development by Thymus-Specific Proteasomes. Science 2007; 316:1349-1353.

18. Kelly RJ, Petrini I, Rajan A, Wang Y and Giaccone G. Thymic Malignancies: From Clinical Management to
Targeted Therapies. J Clin Oncol. 2011; 29:4820-4827.

19. Engels EA. Epidemiology of thymoma and associated malignancies. J Thorac Oncol. 2010; 5:S260-265.

20. Rosai J. (1999). Histological Typing of Tumors of the Thymus. (Berlin, Germany: Spring-Verlag).

21. Okumura M, Ohta M, Tateyama H, Nakagawa K, Matsumura A, Maeda H, Tada H, Eimoto T, Matsuda H and Masaoka A. The World Health Organization histologic classification system reflects the oncologic behavior of thymoma. Cancer. 2002; 94:624-632.

22. Nakagawa K, Asamura H, Matsuno Y, Suzuki K, Kondo H, Maeshima A, Miyaoka E and Tsuchiya R. Thymoma: a clinicopathologic study based on the new World Health Organization classification. J Thorac Cardiovasc Surg. 2003; 126:1134-1140.

23. Moran CA, Weissferdt A, Kalhor N, Solis LM, Behrens C, Wistuba, II and Suster S. Thymomas I: a clinicopathologic correlation of 250 cases with emphasis on the World Health Organization schema. Am J Clin Pathol. 2012; 137:444450.

24. Zhou R, Zettl A, Strobel P, Wagner K, Muller-Hermelink HK, Zhang S, Marx A and Starostik P. Thymic epithelial tumors can develop along two different pathogenetic pathways. Am J Pathol. 2001; 159:1853-1860.

25. Inoue $\mathrm{M}$, Starostik $\mathrm{P}$, Zettl A, Strobel P, Schwarz S, Scaravilli F, Henry K, Willcox N, Muller-Hermelink HK and Marx A. Correlating genetic aberrations with World Health Organization-defined histology and stage across the spectrum of thymomas. Cancer Res. 2003; 63:3708-3715.

26. Kuraguchi M, Wang XP, Bronson RT, Rothenberg R, Ohene-Baah NY, Lund JJ, Kucherlapati M, Maas RL and Kucherlapati R. Adenomatous polyposis coli (APC) is required for normal development of skin and thymus. PLoS Genet. 2006; 2:e146.

27. Aoki $\mathrm{K}$ and Taketo MM. Adenomatous polyposis coli (APC): a multi-functional tumor suppressor gene. J Cell Sci. 2007; 120:3327-3335.

28. Zuklys S, Gill J, Keller MP, Hauri-Hohl M, Zhanybekova S, Balciunaite G, Na K-J, Jeker LT, Hafen K, Tsukamoto N, Amagai T, Taketo MM, Krenger W and Holländer GA. Stabilized beta-Catenin in Thymic Epithelial Cells Blocks Thymus Development and Function. J Immunol. 2009; 182:2997-3007.

29. Lo Celso C, Prowse DM and Watt FM. Transient activation of beta-catenin signalling in adult mouse epidermis is sufficient to induce new hair follicles but continuous activation is required to maintain hair follicle tumours. Development 2004; 131:1787-1799.

30. Van Mater D, Kolligs FT, Dlugosz AA and Fearon ER. Transient activation of beta -catenin signaling in cutaneous keratinocytes is sufficient to trigger the active growth phase of the hair cycle in mice. Gene Dev. 2003; 17:1219-1224.

31. Marx A, Hohenberger P, Hoffmann H, Pfannschmidt J, Schnabel P, Hofmann H-S, Wiebe K, Schalke B, Nix 
W, Gold R, Willcox N, Peterson P and Ströbel P. The Autoimmune Regulator AIRE in Thymoma Biology: Autoimmunity and Beyond. J Thorac Oncol. 2010; 5:S266-S272.

32. Dotto J, Pelosi $G$ and Rosai J. Expression of p63 in thymomas and normal thymus. Am J Clin Pathol. 2007; 127:415-420.

33. Kikuchi T, Ichimiya S, Kojima T, Crisa L, Koshiba S, Tonooka A, Kondo N, Van Der Saag PT, Yokoyama S and Sato N. Expression profiles and functional implications of p53-like transcription factors in thymic epithelial cell subtypes. Int Immunol. 2004; 16:831-841.

34. Chilosi M, Zamo A, Brighenti A, Malpeli G, Montagna L, Piccoli P, Pedron S, Lestani M, Inghirami G, Scarpa A, Doglioni $\mathrm{C}$ and Menestrina F. Constitutive expression of DeltaN-p63alpha isoform in human thymus and thymic epithelial tumours. Virchows Arch. 2003; 443:175-183.

35. Yang A, Kaghad M, Wang Y, Gillett E, Fleming MD, D^tsch V, Andrews NC, Caput D and McKeon F. p63, a p53 Homolog at 3q27 29, Encodes Multiple Products with Transactivating, Death-Inducing, and Dominant-Negative Activities. Mol Cell 1998; 2:305-316.

36. Zhang Z, Li W, Cheng S, Yao H, Zhang F, Chang Q, Ke Z, Wang X, Son YO, Luo J and Shi X. Nickel-induced downregulation of DeltaNp63 and its role in the proliferation of keratinocytes. Toxicol Appl Pharmacol. 2011; 253:235-243.

37. Westfall MD, Mays DJ, Sniezek JC and Pietenpol JA. The Delta Np63 alpha phosphoprotein binds the p21 and 14-3-3 sigma promoters in vivo and has transcriptional repressor activity that is reduced by Hay-Wells syndrome-derived mutations. Mol Cell Biol. 2003; 23:2264-2276.

38. King KE, Ponnamperuma RM, Allen C, Lu H, Duggal P, Chen Z, Van Waes C and Weinberg WC. The p53 homologue DeltaNp63alpha interacts with the nuclear factor-kappaB pathway to modulate epithelial cell growth. Cancer Res. 2008; 68:5122-5131.

39. Petitjean A, Ruptier C, Tribollet V, Hautefeuille A, Chardon F, Cavard C, Puisieux A, Hainaut P and Caron de Fromentel C. Properties of the six isoforms of p63: p53like regulation in response to genotoxic stress and cross talk with deltaNp73. Carcinogenesis. 2008; 29:273-281.

40. Baldi A, Ambrogi V, Mineo D, Mellone P, Campioni M, Citro G and Mineo TC. Analysis of cell cycle regulator proteins in encapsulated thymomas. Clin Cancer Res. 2005; 11:5078-5083.

41. Ripen AM, Nitta T, Murata S, Tanaka K and Takahama Y. Ontogeny of thymic cortical epithelial cells expressing the thymoproteasome subunit beta5t. Eur J Immunol. 2011; 41:1278-1287.

42. Takahama Y, Takada K, Murata S and Tanaka K. beta5tcontaining thymoproteasome: specific expression in thymic cortical epithelial cells and role in positive selection of CD8+ T cells. Curr Opin Immunol. 2012; 24:92-98.

43. Yamada Y, Tomaru U, Ishizu A, Kiuchi T, Marukawa K,
Matsuno Y and Kasahara M. Expression of proteasome subunit beta5t in thymic epithelial tumors. Am J Surg Pathol. 2011; 35:1296-1304.

44. Hayashi A, Fumon T, Miki Y, Sato H, Yoshino T and Takahashi K. The evaluation of immunohistochemical markers and thymic cortical microenvironmental cells in distinguishing thymic carcinoma from type b3 thymoma or lung squamous cell carcinoma. J Clin Exp Hematop. 2013; 53:9-19.

45. Bickenbach JR, Greer JM, Bundman DS, Rothnagel JA and Roop DR. Loricrin expression is coordinated with other epidermal proteins and the appearance of lipid lamellar granules in development. J Invest Dermatol. 1995; 104:405410.

46. Mehrel T, Hohl D, Rothnagel JA, Longley MA, Bundman D, Cheng C, Lichti U, Bisher ME, Steven AC, Steinert $\mathrm{PM}$ and et al. Identification of a major keratinocyte cell envelope protein, loricrin. Cell. 1990; 61:1103-1112.

47. Strobel P, Hohenberger P and Marx A. Thymoma and thymic carcinoma: molecular pathology and targeted therapy. J Thorac Oncol. 2010; 5:S286-290.

48. Park W-Y, Kimb J-I, Shim E-H, Lee W-H, Kim S-H, Seo J-W, Jang J-J and Seoab J-S. Development of thymic carcinoma in transgenic mice expressing SV40 T antigen. Cancer Lett. 1996; 107:293-300.

49. Carraresi L, Tripodi SA, Mulder LC, Bertini S, Nuti S, Schuerfeld K, Cintorino M, Bensi G, Rossini M and Mora M. Thymic hyperplasia and lung carcinomas in a line of mice transgenic for keratin 5-driven HPV16 E6/E7 oncogenes. Oncogene. 2001; 20:8148-8153.

50. Weinberg RA. (2007). The biology of cancer. (New York: Garland Science, Taylor and Francis Group).

51. Harbour JW, Luo RX, Santi AD, Postigo AA and Dean DC. Cdk Phosphorylation Triggers Sequential Intramolecular Interactions that Progressively Block Rb Functions as Cells Move through G1. Cell. 1999; 98:859-869.

52. Scheijen B, Bronk M, van der Meer T, De Jong D and Bernards R. High Incidence of Thymic Epithelial Tumors in E2F2 Transgenic Mice. J Biol Chem. 2004; 279:1047610483.

53. Moriyama A, Kii I, Sunabori T, Kurihara S, Takayama I, Shimazaki M, Tanabe H, Oginuma M, Fukayama M, Matsuzaki Y, Saga Y and Kudo A. GFP transgenic mice reveal active canonical Wnt signal in neonatal brain and in adult liver and spleen. Genesis. 2007; 45:90-100.

54. Liang C-C, You LR, Chang J-L, Tsai T-F and Chen C-M. Transgenic mice exhibiting inducible and spontaneous Cre activities driven by a bovine keratin 5 promoter that can be used for the conditional analysis of basal epithelial cells in multiple organs. J Biomed Sci. 2009; 16:2.

55. Brault V, Moore R, Kutsch S, Ishibashi M, Rowitch DH, McMahon AP, Sommer L, Boussadia O and Kemler R. Inactivation of the beta-catenin gene by Wnt1-Cre-mediated deletion results in dramatic brain malformation and failure 
of craniofacial development. Development 2001; 128:12531264.

56. Lu T-L, Huang Y-F, You L-R, Chao N-C, Su F-Y, Chang $\mathrm{J}-\mathrm{L}$ and Chen C-M. Conditionally Ablated Pten in Prostate Basal Cells Promotes Basal-to-Luminal Differentiation and Causes Invasive Prostate Cancer in Mice. Am J Pathol. 2013; 182:975-991.

57. Chen C-M and Behringer RR. Ovcal regulates cell proliferation, embryonic development, and tumorigenesis. Gene Dev. 2004; 18:320-332.

58. Lu T-L, Chang J-L, Liang C-C, You L-R and Chen C-M. Tumor Spectrum, Tumor Latency and Tumor Incidence of the Pten-Deficient Mice. PLoS ONE. 2007; 2:e1237.

59. Chen C-M, Wang H-Y, You L-R, Shang R-L and Liu F-C. Expression analysis of an evolutionary conserved metallophosphodiesterase gene, Mpped1, in the normal and beta-catenin-deficient malformed dorsal telencephalon. Dev Dyn. 2010; 239:1797-1806.

60. Lu T-L and Chen C-M. Differential Requirements for $\beta$-Catenin in Murine Prostate Cancer Originating from Basal versus Luminal Cells. J Pathol. 2015; 236:290-301. 